\title{
Probabilistic Representation of Weak Solutions of Partial Differential Equations with Polynomial Growth Coefficients*
}

\author{
Qi Zhang ${ }^{\text {a }}$, Huaizhong Zhao ${ }^{\text {b }}$
}

\begin{abstract}
I
$\mathrm{n}$ this paper we develop a new weak convergence and compact embedding method to study the existence and uniqueness of the $L_{\rho}^{2 p}\left(\mathbb{R}^{d} ; \mathbb{R}^{1}\right) \times L_{\rho}^{2}\left(\mathbb{R}^{d} ; \mathbb{R}^{d}\right)$ valued solution of backward stochastic differential equations with p-growth coefficients. Then we establish the probabilistic representation of the weak solution of PDEs with p-growth coefficients via corresponding BSDEs.

Keywords: PDEs with polynomial growth coefficients, generalized Feynman-Kac formula, probabilistic representation of weak solutions, backward stochastic differential equations, weak convergence, compact embedding.
\end{abstract}

AMS 2000 subject classifications: 60H10, 60H30, 35K55.

\section{Introduction}

In this paper, we study the probabilistic representation of the weak solution of a class of parabolic partial differential equations (PDEs) on $\mathbb{R}^{d}$ with p-growth coefficients

$$
\left\{\begin{array}{l}
\frac{\partial v}{\partial t}(t, x)=\mathscr{L} v(t, x)+f\left(x, v(t, x),\left(\sigma^{*} \nabla v\right)(t, x)\right), \quad 0 \leq t \leq T, \\
v(0, x)=h(x),
\end{array}\right.
$$

by the solution of the corresponding backward stochastic differential equations (BSDEs) in $\rho$-weighted $L^{2}$ space. Here $\mathscr{L}$ is a second order differential operator

$$
\mathscr{L}=\frac{1}{2} \sum_{i, j=1}^{d} a_{i j}(x) \frac{\partial^{2}}{\partial x_{i} \partial x_{j}}+\sum_{i=1}^{d} b_{i}(x) \frac{\partial}{\partial x_{i}},
$$

$\left(a_{i j}(x)\right)$ is a symmetric matrix with a decomposition $\left(a_{i j}(x)\right)=\left(\sigma_{i j}(x)\right)\left(\sigma_{i j}(x)\right)^{*}, f:(x, y, z) \mapsto$ $f(x, y, z)$ is a function of polynomial growth in $y$ and Lipschitz continuous in $z$. Many partial differ-

a School of Mathematical Sciences, Fudan University, Shanghai, 200433, China.

b Department of Mathematical Sciences, Loughborough University, Loughborough, LE11 3TU, UK.

Emails: qzh@fudan.edu.cn; H.Zhao@lboro.ac.uk

* Accepted by Journal of Theoretical Probability, to appear. DOI: 10.1007/s10959-011-0350-y 
ential equations arising in physics, engineering and biology have polynomial growth nonlinear terms e.g. KPP-Fisher equations, Allen-Cahn equations and Ginzburg-Landau equations. The representation provides an important connection between stochastic flows generated by $\mathscr{L}$ and the weak solutions of PDEs possibly with polynomial growth coefficients. In connection with the classical solutions of the linear parabolic PDEs, the well-known Feynman-Kac formula provides the probabilistic representation for them and originated many important developments (Feynman [8], Kac [12]). An alternative probabilistic representation using only the values of a finite (random) set of times to the linear heat equations was obtained recently by Dalang, Mueller and Tribe [4. This idea made it possible for them to obtain corresponding formula for a wide class of linear PDEs such as some wave equations with potentials. The Feynman-Kac formula has played important roles in problems such as the large deviation theory of Donsker and Varadhan [6, Wentzell and Freidlin 24, small time asymptotics of heat kernel and its logarithmic derivatives, in particular on Riemannian manifolds (Elworthy [10, Malliavin and Stroock [16]). The Feynman-Kac formula has been extended and used to quasi-linear parabolic type partial differential equations, especially, in the study of the generalized KPP equations using the large deviation theory method by Freidlin [9], using the semi-classical probabilistic method by Elworthy, Truman and Zhao [7. The study of the quasi-linear parabolic type PDE is based on an equation of the Feynman-Kac type integration of stochastic functionals. The approach of the backward stochastic differential equations, pioneered by Pardoux and Peng [19], [20] originally, provided an alternative approach to the classical solution of the parabolic type PDEs, when the coefficients of the PDE are sufficiently regular and Lipschitz continuous. This was extended to the viscosity solution of a large class of partial differential equations and BSDEs. They include the linear growth case considered by Lepeltier and San Martin [15, the quadratic coefficients (in $z$ ) considered by Kobylanski [13, Briand and $\mathrm{Hu}$ [3], and the polynomial growth coefficients in Pardoux [18]. The solution of the BSDEs in above cases gives the probabilistic representation of the classical or viscosity solution of the PDEs as a generalization to the Feynman-Kac formula. Applications of BSDEs have been found in some problems such as a model in mathematics of finance (El Karoui, Peng and Quenez [11]), as an efficient method for constructing $\Gamma$-martingales on Riemannian manifolds (Darling [5]), and as an intrinsic tool to construct the pathwise stationary solution for stochastic PDEs (Zhang and Zhao [25], 26]).

The Feynman-Kac approach to a Sobolev or $L^{2}$ space valued weak solution of PDEs has been concentrated mainly on linear problems. Many important progress has been made e.g. in quantum field theory (see 22]). The probabilistic approach to the weak solution of quasi-linear PDEs stayed behind. Regularity of the solutions, even in the sense of weak derivative, was not given in Freidlin's probabilistic approach of generalized solution formally represented by the Feynman-Kac formula ([9]). The BSDEs start to show some usefulness in this aspect, when the coefficients are of Lipschitz continuous in the space $L_{\rho}^{2}\left(\mathbb{R}^{d} ; \mathbb{R}^{1}\right) \times L_{\rho}^{2}\left(\mathbb{R}^{d} ; \mathbb{R}^{d}\right)$ or of linear growth, and monotone, from the work of Barles and Lesigne [2, Bally and Matoussi [1, Zhang and Zhao [25, 26]. The objective of this paper is to move away from the assumption of the linear growth of $f$ and from considering the classical or viscosity solution of PDEs to establish the probabilistic representation for the weak solution of such polynomial growth PDEs. Although the connection of BSDEs with the viscosity solution for the cases of quadratic and polynomial growth has been obtained in [13, [18 respectively, the existing methods in the study of BSDEs for finding the solution of the BSDEs in $L_{\rho}^{2}\left(\mathbb{R}^{d} ; \mathbb{R}^{1}\right) \times L_{\rho}^{2}\left(\mathbb{R}^{d} ; \mathbb{R}^{d}\right)$ are not adequate to solve the problem of the weak solution of BSDEs with p-growth coefficients. The fixed point method in $M^{2}\left([t, T] ; L_{\rho}^{2}\left(\mathbb{R}^{d} ; \mathbb{R}^{1}\right)\right) \times M^{2}\left([t, T] ; L_{\rho}^{2}\left(\mathbb{R}^{d} ; \mathbb{R}^{d}\right)\right)$, which is equivalent to finding a strongly convergent sequence in the same space, seems difficult to work for the problem with p-growth coefficients. It is 
also inadequate to use a combination of the weak convergence in finite dimensional space developed by Pardoux [18] and the weak solution method developed by Bally and Matoussi [1, Zhang and Zhao [25], 26], to solve this problem. We need to introduce some new ideas to the study of BSDEs. The progress of this problem was made when we realized that, in addition to the method of Zhang and Zhao ([25], 26]), as well as the standard approach using Alaoglu lemma to find a weakly convergent sequence $\left(Y^{n}, Z^{n}\right)$, we can use the equivalence of norm principle and Rellich-Kondrachov Compactness Theorem to get a strongly convergent sequence $Y^{n}$. Our recent result on the $S^{2}\left([t, T], L_{\rho}^{2}\left(\mathbb{R}^{d} ; \mathbb{R}^{1}\right)\right) \times$ $M^{2}\left([t, T] ; L_{\rho}^{2}\left(\mathbb{R}^{d} ; \mathbb{R}^{d}\right)\right)$ valued solution of BDSDEs with non-Lipschitz linear growth coefficients made it possible for us to study the BSDEs in $S^{2 p}\left([t, T], L_{\rho}^{2 p}\left(\mathbb{R}^{d} ; \mathbb{R}^{1}\right)\right) \times M^{2}\left([t, T] ; L_{\rho}^{2}\left(\mathbb{R}^{d} ; \mathbb{R}^{d}\right)\right)$ with polynomial growth coefficients, even without assuming $f$ being locally Lipschitz continuous in $y$. Of course, we need to assume the monotonicity condition of $f$ in $y$. Moreover, it is also an essential step to prove the strong convergence of $Z^{n}$ in $M^{2}\left([t, T], L_{\rho}^{2}\left(\mathbb{R}^{d} ; \mathbb{R}^{d}\right)\right)$ from the result of the strong convergence of $Y^{n}$ and Itô's formula. The weak convergence and compact embedding method has been used in the study of PDEs. However, as far as we know, to use this kind of argument to the study of BSDE, this paper is the first time in literature. The equivalence of norm principle and very careful probabilistic (measure theoretical) and analytic arguments including localization made it work in the probabilistic context. However, the probabilistic case is a lot more complicated than the deterministic PDEs case as we need to work on the space $\Omega \times[0, T] \times \mathbb{R}^{d}$ and solve the equation with probability one, instead only work on $[0, T] \times \mathbb{R}^{d}$ in the deterministic PDEs case. The probabilistic representation can be regarded as a generalized Feynman-Kac formula to the weak solution of the PDEs with p-growth coefficients. We would like to point out that analysts already studied PDE with polynomial growth coefficients when these coefficients do not depend on $\nabla u$ (see Robinson [21], Temam [23]). Our method pushes the study to more general equation with nonlinear term depending on $\nabla u$. But this is not the main purpose of the paper. Our main purpose is to find a method to solve the BSDEs in $L_{\rho}^{2 p}\left(\mathbb{R}^{d} ; \mathbb{R}^{1}\right) \times L_{\rho}^{2}\left(\mathbb{R}^{d} ; \mathbb{R}^{d}\right)$ space with polynomial growth coefficients, so it provides a probabilistic representation to the corresponding PDEs. This is new in literature. Moreover, our approach does not depend on results of PDEs. Rather we can obtain results about PDEs from the study of BSDEs. Due to this important aspect of our results here, we can extend this result to backward doubly stochastic differential equations (BDSDEs) so that we obtain new results on the stationary solutions of SPDEs via BDSDEs with polynomial growth coefficients. See Zhang and Zhao [27. We believe our method will be useful to other types of PDEs or SPDEs and BSDEs or BDSDEs as well.

After this paper was completed, we were informed the paper Matoussi and Xu [17. But we would like to point out what we have proved as well as our methods are different. Notice the convergence $\left(Y_{s}^{t, x, n}, Z_{s}^{t, x, n}\right)$ is only a weak convergence along a subsequence according to the Alaoglu lemma. If one considers weak convergence in $\left.M^{2}\left([t, T], \mathbb{R}^{1}\right) \times M^{2}\left([t, T] ; \mathbb{R}^{d}\right)\right)$, which worked well in Pardoux [18] for the case of viscosity solutions of the PDEs, then each weak convergence is for a fixed $x$, and the choice of subsequence may depend on $x$. However, this will cause serious problems when one considers weak solutions. Our approach to avoid this essential difficulty is to find a subsequence of the weak convergence in the space $M^{2}\left([t, T], L_{\rho}^{2}\left(\mathbb{R}^{d} ; \mathbb{R}^{1}\right)\right) \times M^{2}\left([t, T] ; L_{\rho}^{2}\left(\mathbb{R}^{d} ; \mathbb{R}^{d}\right)\right)$. The whole point and major difficulty of this approach are to pass the limit term by term in the approximating equation to the desired limit. This is achieved in our paper by obtaining a strong convergent subsequence of $\left(Y_{s}^{t, x, n}, Z_{s}^{t, x, n}\right)$ in $M^{2}\left([t, T], L_{\rho}^{2}\left(\mathbb{R}^{d} ; \mathbb{R}^{1}\right)\right) \times M^{2}\left([t, T] ; L_{\rho}^{2}\left(\mathbb{R}^{d} ; \mathbb{R}^{d}\right)\right)$ using the Rellich-Kondrachov compactness theorem and generalized equivalence of norm principle as we have already mentioned. 


\section{The main results}

In this paper, we study the weak solutions of a class of parabolic PDEs with p-growth coefficients, their corresponding backward stochastic differential equations (BSDEs) in a Hilbert space ( $\rho$-weighted $L^{2}$ space) and the probabilistic representation of the weak solutions of (1.1) by using the solutions of BSDEs. For an arbitrary fixed $t \in[0, T]$, we start from the following SDE:

$$
X_{s}^{t, x}=x+\int_{t}^{s} b\left(X_{r}^{t, x}\right) d r+\int_{t}^{s} \sigma\left(X_{r}^{t, x}\right) d W_{r}, \quad s \geq t
$$

where $W$ is a $\mathbb{R}^{d}$ Brownian motion on a probability space $(\Omega, \mathscr{F}, P)$, and $b: \mathbb{R}^{d} \rightarrow \mathbb{R}^{d}, \sigma: \mathbb{R}^{d} \rightarrow \mathbb{R}^{d \times d}$ are measurable. For given $t \in[0, T]$ in (2.1), we consider a slightly more general BSDEs for $s \geq t$ by allowing $f$ depending on time explicitly:

$$
Y_{s}^{t, x}=h\left(X_{T}^{t, x}\right)+\int_{s}^{T} f\left(r, X_{r}^{t, x}, Y_{r}^{t, x}, Z_{r}^{t, x}\right) d r-\int_{s}^{T}\left\langle Z_{r}^{t, x}, d W_{r}\right\rangle,
$$

where $f:[0, T] \times \mathbb{R}^{d} \times \mathbb{R}^{1} \times \mathbb{R}^{d} \rightarrow \mathbb{R}^{1}$ and $h: \mathbb{R}^{d} \rightarrow \mathbb{R}^{1}$ are measurable. More conditions on $b, \sigma, f$ are needed and will be specified later. The Hilbert space $L_{\rho}^{2}\left(\mathbb{R}^{d} ; \mathbb{R}^{k}\right)$ is the space containing all Borel measurable functions $l: \mathbb{R}^{d} \rightarrow \mathbb{R}^{k}$ such that $\int_{\mathbb{R}^{d}}<l(x), l(x)>\rho^{-1}(x) d x<\infty$, with the inner product

$$
\left\langle u_{1}, u_{2}\right\rangle=\int_{\mathbb{R}^{d}}<u_{1}(x), u_{2}(x)>\rho^{-1}(x) d x
$$

where $\rho(x)=(1+|x|)^{q}, q>d$, is a weight function. The Banach space $L_{\rho}^{2 p}\left(\mathbb{R}^{d} ; \mathbb{R}^{1}\right)$ is the space containing all Borel measurable functions $l: \mathbb{R}^{d} \rightarrow \mathbb{R}^{1}$ such that $\int_{\mathbb{R}^{d}} l^{2 p}(x) \rho^{-1}(x) d x<\infty$ with the norm $\|l\|_{L_{\rho}^{2 p}\left(R^{d}\right)}=\left(\int_{\mathbb{R}^{d}} l^{2 p}(x) \rho^{-1}(x) d x\right)^{\frac{1}{2 p}}$. It is easy to see that $\rho(x): \mathbb{R}^{d} \longrightarrow \mathbb{R}^{1}$ is a continuous positive function satisfying $\int_{\mathbb{R}^{d}} \rho^{-1}(x) d x<\infty$. Note that we can consider more general $\rho$ which satisfies the above condition and conditions in [1] and all the results of this paper still hold. For $k \geq 0$, we denote by $C_{b}^{k}$ the set of $C^{k}$-functions whose partial derivatives of order less than or equal to $k$ are bounded, and denote by $H_{\rho}^{1}$ the $\rho$-weighted Sobolev space, i.e. the completion of $C_{c}^{\infty}\left(\mathbb{R}^{d} ; \mathbb{R}^{1}\right)$ w.r.t. the norm $\|\varphi\|_{H_{\rho}^{1}\left(\mathbb{R}^{d} ; \mathbb{R}^{1}\right)}^{2}=\int_{\mathbb{R}^{d}}\left(|\varphi(x)|^{2}+|\nabla \varphi(x)|^{2}\right) \rho^{-1}(x) d x$. Now we assume the following conditions for the coefficients in SDE (2.1) and BSDE (2.2):

(H.1). For a given $p \geq 1, \int_{\mathbb{R}^{d}}|h(x)|^{2 p} \rho^{-1}(x) d x<\infty$.

(H.2). There exists a constant $C \geq 0$ and a function $f_{0}$ with $\int_{0}^{T} \int_{\mathbb{R}^{d}}\left|f_{0}(s, x)\right|^{2 p} \rho^{-1}(x) d x d s<\infty$ s.t. $|f(s, x, y, z)| \leq C\left(\left|f_{0}(s, x)\right|+|y|^{p}+|z|\right)$, where $p$ is the same as in (H.1).

(H.3). There exists a constant $\mu \in \mathbb{R}^{1}$ s.t. for any $s \in[0, T], y_{1}, y_{2} \in \mathbb{R}^{1}, x, z \in \mathbb{R}^{d}$,

$$
\left(y_{1}-y_{2}\right)\left(f\left(s, x, y_{1}, z\right)-f\left(s, x, y_{2}, z\right)\right) \leq \mu\left|y_{1}-y_{2}\right|^{2} .
$$

(H.4). The function $(y, z) \rightarrow f(s, x, y, z)$ is continuous and $z \rightarrow f(s, x, y, z)$ is globally Lipschitz continuous with Lipschitz constant $L \geq 0$, i.e. for any $s \in[0, T], y \in \mathbb{R}^{1}, x, z_{1}, z_{2} \in \mathbb{R}^{d}$,

$$
\left|f\left(s, x, y, z_{1}\right)-f\left(s, x, y, z_{2}\right)\right| \leq L\left|z_{1}-z_{2}\right| .
$$

(H.5). The coefficients $b \in C_{b}^{2}\left(\mathbb{R}^{d} ; \mathbb{R}^{d}\right), \sigma \in C_{b}^{3}\left(\mathbb{R}^{d} ; \mathbb{R}^{d} \times \mathbb{R}^{d}\right)$ and $\sigma$ satisfies the uniform ellipticity condition, i.e. there exists a constant $D>0$ s.t. $\xi^{*}\left(\sigma \sigma^{*}\right)(x) \xi \geq D \xi^{*} \xi$ for any $\xi \in \mathbb{R}^{d}$. 
Condition (H.5) guarantees the existence of the flow of diffeomorphism. This is essential in the equivalence of norm principle (Lemma 3.2), which together with the uniform ellipticity condition implies the equivalence of the Sobolev norm of the solution of the PDE and $L^{2}\left(\mathbb{R}^{d} ; \mathbb{R}^{1}\right) \times L^{2}\left(\mathbb{R}^{d} ; \mathbb{R}^{d}\right)$ norm of the solution of the BSDE. See (4.2) in Section 4.

It is easy to see that for a.a. $x \in \mathbb{R}^{d},\left(Y_{s}^{t, x}, Z_{s}^{t, x}\right)$ solves BSDE (2.2) if and only if $\left(\tilde{Y}_{s}^{t, x}, \tilde{Z}_{s}^{t, x}\right)=$ $\left(\mathrm{e}^{\mu s} Y_{s}^{t, x}, \mathrm{e}^{\mu s} Z_{s}^{t, x}\right)$ solves the following BSDE:

$$
\tilde{Y}_{s}^{t, x}=\mathrm{e}^{\mu T} h\left(X_{T}^{t, x}\right)+\int_{s}^{T} \tilde{f}\left(r, X_{r}^{t, x}, \tilde{Y}_{r}^{t, x}, \tilde{Z}_{r}^{t, x}\right) d r-\int_{s}^{T}\left\langle\tilde{Z}_{r}^{t, x}, d W_{r}\right\rangle,
$$

where $\tilde{f}(r, x, y, z)=\mathrm{e}^{\mu r} f\left(r, x, \mathrm{e}^{-\mu r} y, \mathrm{e}^{-\mu r} z\right)-\mu y$. We can verify that $\tilde{f}$ satisfies Conditions (H.2), (H.3) and (H.4). But, by Condition (H.3), for $y_{1}, y_{2} \in \mathbb{R}^{1}$, and $x, z \in \mathbb{R}^{d}$,

$$
\begin{aligned}
& \left(y_{1}-y_{2}\right)\left(\tilde{f}\left(s, x, y_{1}, z\right)-\tilde{f}\left(s, x, y_{2}, z\right)\right) \\
= & \mathrm{e}^{2 \mu s}\left(\mathrm{e}^{-\mu s} y_{1}-\mathrm{e}^{-\mu s} y_{2}\right)\left(f\left(s, x, \mathrm{e}^{-\mu s} y_{1}, \mathrm{e}^{-\mu s} z\right)-f\left(s, x, \mathrm{e}^{-\mu s} y_{2}, \mathrm{e}^{-\mu s} z\right)\right)-\mu\left(y_{1}-y_{2}\right)\left(y_{1}-y_{2}\right) \\
\leq & \mu \mathrm{e}^{2 \mu s}\left|\mathrm{e}^{-\mu s} y_{1}-\mathrm{e}^{-\mu s} y_{2}\right|^{2}-\mu\left|y_{1}-y_{2}\right|^{2}=0 .
\end{aligned}
$$

Now we give the definition for the solution of BSDE (2.2) in the $\rho$-weighted $L^{2}$ space. First define the space for the solution $\left(Y^{t, \cdot}, Z^{t, \cdot}\right)$. We denote by $\mathcal{N}$ the class of $P$-null sets of $\mathscr{F}$ and let $\mathscr{F}_{t} \triangleq \mathscr{F}_{t}^{W} \bigvee \mathcal{N}$, for $0 \leq t \leq T$. We recall some definitions.

Definition 2.1 (Definitions 2.2 in [25]) Let $\mathbb{S}$ be a separable Banach space with norm $\|\cdot\|_{\mathbb{S}}$ and Borel $\sigma$-field $\mathscr{S}$ and $q \geq 2$ be a real number. We denote by $M^{q}([t, T] ; \mathbb{S})$ the set of $\mathscr{B}([t, T]) \otimes \mathscr{F} / \mathscr{S}$ measurable random processes $\{\phi(s)\}_{t \leq s \leq T}$ with values in $\mathbb{S}$ satisfying

(i) $\phi(s): \Omega \rightarrow \mathbb{S}$ is $\mathscr{F}_{s}$ measurable for $t \leq s \leq T$;

(ii) $E\left[\int_{t}^{T}\|\phi(s)\|_{\mathbb{S}}^{q} d s\right]<\infty$.

Also we denote by $S^{q}([t, T] ; \mathbb{S})$ the set of $\mathscr{B}([t, T]) \otimes \mathscr{F} / \mathscr{S}$ measurable random processes $\{\psi(s)\}_{t \leq s \leq T}$ with values in $\mathbb{S}$ satisfying

(i) $\psi(s): \Omega \rightarrow \mathbb{S}$ is $\mathscr{F}_{s}$ measurable for $t \leq s \leq T$ and $\psi(\cdot, \omega)$ is continuous $P$-a.s.;

(ii) $E\left[\sup _{t \leq s \leq T}\|\psi(s)\|_{\mathbb{S}}^{q}\right]<\infty$.

Definition 2.2 (Definitions 3.1 in [25]) A pair of processes $\left(Y_{s}^{t, x}, Z_{s}^{t, x}\right)$ is called a solution of BSDE (2.2) if $\left(Y^{t, \cdot}, Z^{t, \cdot}\right) \in S^{2 p}\left([t, T] ; L_{\rho}^{2 p}\left(\mathbb{R}^{d} ; \mathbb{R}^{1}\right)\right) \times M^{2}\left([t, T] ; L_{\rho}^{2}\left(\mathbb{R}^{d} ; \mathbb{R}^{d}\right)\right)$ and $\left(Y_{s}^{t, x}, Z_{s}^{t, x}\right)$ satisfies (2.2) for a.a. $x$, with probability one.

Remark 2.3 Due to the density of $C_{c}^{0}\left(\mathbb{R}^{d} ; \mathbb{R}^{1}\right)$ in $L_{\rho}^{2}\left(\mathbb{R}^{d} ; \mathbb{R}^{d}\right),\left(Y_{s}^{t, x}, Z_{s}^{t, x}\right)$ satisfies (2.2) for a.a. $x$ with probability one is equivalent to that for an arbitrary $\varphi \in C_{c}^{0}\left(\mathbb{R}^{d} ; \mathbb{R}^{1}\right),\left(Y_{s}^{t, x}, Z_{s}^{t, x}\right)$ satisfies

$$
\begin{aligned}
\int_{\mathbb{R}^{d}} Y_{s}^{t, x} \varphi(x) d x= & \int_{\mathbb{R}^{d}} h\left(X_{T}^{t, x}\right) \varphi(x) d x+\int_{s}^{T} \int_{\mathbb{R}^{d}} f\left(r, X_{r}^{t, x}, Y_{r}^{t, x}, Z_{r}^{t, x}\right) \varphi(x) d x d r \\
& -\int_{s}^{T}\left\langle\int_{\mathbb{R}^{d}} Z_{r}^{t, x} \varphi(x) d x, d W_{r}\right\rangle \quad P-\text { a.s. }
\end{aligned}
$$

Since $\left(Y^{t, \cdot}, Z^{t, \cdot}\right) \in S^{2 p}\left([t, T] ; L_{\rho}^{2 p}\left(\mathbb{R}^{d} ; \mathbb{R}^{1}\right)\right) \times M^{2}\left([t, T] ; L_{\rho}^{2}\left(\mathbb{R}^{d} ; \mathbb{R}^{d}\right)\right)$ if and only if $\left(\tilde{Y}^{t, \cdot}, \tilde{Z}^{t, \cdot}\right) \in$ $S^{2 p}\left([t, T] ; L_{\rho}^{2 p}\left(\mathbb{R}^{d} ; \mathbb{R}^{1}\right)\right) \times M^{2}\left([t, T] ; L_{\rho}^{2}\left(\mathbb{R}^{d} ; \mathbb{R}^{d}\right)\right)$, so we claim $\left(Y_{s}^{t, x}, Z_{s}^{t, x}\right)$ is the solution of BSDE (2.2) 
in the $\rho$-weighted $L^{2}$ space if and only if $\left(\tilde{Y}_{s}^{t, x}, \tilde{Z}_{s}^{t, x}\right)$ is the solution of BSDE (2.3) in $\rho$-weighted $L^{2}$ space. Therefore we can replace, without losing any generality, Condition (H.3) by

$(\text { H.3 })^{*}$. For any $s \in[0, T], y_{1}, y_{2} \in \mathbb{R}^{1}, x, z \in \mathbb{R}^{d}$,

$$
\left(y_{1}-y_{2}\right)\left(f\left(s, x, y_{1}, z\right)-f\left(s, x, y_{2}, z\right)\right) \leq 0 .
$$

The main purpose of this paper is to prove the following two theorems. The first one is about the existence and uniqueness of solutions to BSDE (2.2):

Theorem 2.4 Under Conditions (H.1), (H.2), (H.3)*, (H.4) and (H.5), BSDE (2.2) has a unique solution $\left(Y^{t, \cdot}, Z^{t, \cdot}\right) \in S^{2 p}\left([t, T] ; L_{\rho}^{2 p}\left(\mathbb{R}^{d} ; \mathbb{R}^{1}\right)\right) \times M^{2}\left([t, T] ; L_{\rho}^{2}\left(\mathbb{R}^{d} ; \mathbb{R}^{d}\right)\right)$.

We will establish a connection between BSDE (2.2) and the following PDE with p-growth coefficients:

$$
\left\{\begin{array}{l}
\frac{\partial u}{\partial t}(t, x)=-\mathscr{L} u(t, x)-f\left(t, x, u(t, x),\left(\sigma^{*} \nabla u\right)(s, x)\right), \quad 0 \leq t \leq T, \\
u(T, x)=h(x) .
\end{array}\right.
$$

Noticing $f$ is of p-growth on $y$, we recall the definition for the weak solution of PDE (2.4):

Definition 2.5 Function $u$ is called the weak solution of PDE [2.4) if $\left(u, \sigma^{*} \nabla u\right) \in L^{2 p}\left([0, T] ; L_{\rho}^{2 p}\left(\mathbb{R}^{d} ; \mathbb{R}^{1}\right)\right)$ $\times L^{2}\left([0, T] ; L_{\rho}^{2}\left(\mathbb{R}^{d} ; \mathbb{R}^{d}\right)\right)$ and for an arbitrary $\varphi \in C_{c}^{\infty}\left(\mathbb{R}^{d} ; \mathbb{R}^{1}\right)$,

$$
\begin{aligned}
& \int_{\mathbb{R}^{d}} u(t, x) \varphi(x) d x-\int_{\mathbb{R}^{d}} u(T, x) \varphi(x) d x-\frac{1}{2} \int_{t}^{T} \int_{\mathbb{R}^{d}}\left(\left(\sigma^{*} \nabla u\right)(s, x)\right)^{*}\left(\sigma^{*} \nabla \varphi\right)(x) d x d s \\
& -\int_{t}^{T} \int_{\mathbb{R}^{d}} u(s, x) \operatorname{div}((b-\tilde{A}) \varphi)(x) d x d s \\
= & \int_{t}^{T} \int_{\mathbb{R}^{d}} f\left(s, x, u(s, x),\left(\sigma^{*} \nabla u\right)(s, x)\right) \varphi(x) d x d s, \quad t \in[0, T] .
\end{aligned}
$$

Here $\tilde{A}_{j} \triangleq \frac{1}{2} \sum_{i=1}^{d} \frac{\partial a_{i j}(x)}{\partial x_{i}}$, and $\tilde{A}=\left(\tilde{A}_{1}, \tilde{A}_{2}, \cdots, \tilde{A}_{d}\right)^{*}$.

The other main theorem is the probabilistic representation of PDE (2.4) in the $\rho$-weighted $L^{2}$ space through its corresponding BSDE:

Theorem 2.6 Define $u(t, x)=Y_{t}^{t, x}$, where $\left(Y_{s}^{t, x}, Z_{s}^{t, x}\right)$ is the solution of BSDE (2.2) under Conditions (H.1), (H.2), (H.3)*, (H.4) and (H.5), then u(t,x) is the unique weak solution of PDE 2.4.). Moreover, let $u$ be a representative in the equivalence class of the solution of the PDE 2.4 in $L^{2 p}\left([0, T] ; L_{\rho}^{2 p}\left(\mathbb{R}^{d} ; \mathbb{R}^{1}\right)\right)$ with $\sigma^{*} \nabla u \in L^{2}\left([0, T] ; L_{\rho}^{2}\left(\mathbb{R}^{d} ; \mathbb{R}^{d}\right)\right)$, then $u(t, x)=Y_{t}^{t, x}$ for a.a. $t \in[0, T]$, a.a. $x \in \mathbb{R}^{d}$ and

$$
u\left(s, X_{s}^{t, x}\right)=Y_{s}^{t, x},\left(\sigma^{*} \nabla u\right)\left(s, X_{s}^{t, x}\right)=Z_{s}^{t, x} \text { for a.a. } s \in[t, T], \text { a.a. } x \in \mathbb{R}^{d} \text { a.s. }
$$

We give the proofs of these two theorems in the latter sections.

In Sections 3-5, by making use of truncated BSDEs, we first deal with BSDE (2.2). To prove BSDE (2.2) has a unique solution, we use the Alaoglu lemma to derive a weakly convergent sequence in Section 3 and further use the equivalence of norm principle and Rellich-Kondrachov Compactness Theorem to get a strongly convergent sequence in Section 4. Then we complete the proofs of Theorem 2.4 in Section 5 and consider the corresponding PDE (2.4) to obtain Theorem 2.6 in Section 6 which gives the probabilistic representation to the weak solution of PDE (2.4). 
Remark 2.7 Let $u$ be the weak solution of PDE 2.4) with coefficient $f\left(x, u,\left(\sigma^{*} \nabla u\right)\right)$ which is independent of $t$, we can see easily that $v(t) \triangleq u(T-t)$ is the unique weak solution of PDE (1.1).

\section{The weak convergence}

Assume $f$ satisfies Conditions (H.2), (H.3) ${ }^{*}$ and (H.4). We first use a standard cut-off technique to study a sequence of BSDEs with nonlinear function $f_{n}$ satisfying the linear growth condition on $y$. The $S^{2 p}\left([t, T] ; L_{\rho}^{2 p}\left(\mathbb{R}^{d} ; \mathbb{R}^{1}\right)\right) \times M^{2}\left([t, T] ; L_{\rho}^{2}\left(\mathbb{R}^{d} ; \mathbb{R}^{d}\right)\right)$ valued solution for this kind equation was studied in [26]. For this, we define for each $n \in N$

$$
f_{n}(s, x, y, z)=f\left(s, x, \Pi_{n}(y), z\right),
$$

where $\Pi_{n}(y)=\frac{\inf (n,|y|)}{|y|} y$. Then $f_{n}:[0, T] \times \mathbb{R}^{d} \times \mathbb{R}^{1} \times \mathbb{R}^{d} \rightarrow \mathbb{R}^{1}$ satisfies

(H.2)' For any $s \in[0, T], y \in \mathbb{R}^{1}, x, z \in \mathbb{R}^{d}$ and the constant $C$ given in (H.2),

$$
\left|f_{n}(s, x, y, z)\right| \leq C\left(\left|f_{0}(s, x)\right|+|n|^{p}+|z|\right) .
$$

(H.3)'. For any $s \in[0, T], y_{1}, y_{2} \in \mathbb{R}^{1}, x \in \mathbb{R}^{d}$,

$$
\left(y_{1}-y_{2}\right)\left(f_{n}\left(s, x, y_{1}, z\right)-f_{n}\left(s, x, y_{2}, z\right)\right) \leq 0 .
$$

(H.4)'. The function $(y, z) \rightarrow f_{n}(s, x, y, z)$ is continuous, and for any $s \in[0, T], y \in \mathbb{R}^{1}, x, z_{1}, z_{2} \in \mathbb{R}^{d}$ and the constant $L$ given in (H.4),

$$
\left|f_{n}\left(s, x, y, z_{1}\right)-f_{n}\left(s, x, y, z_{2}\right)\right| \leq L\left|z_{1}-z_{2}\right| .
$$

To see $(\mathrm{H} .3)^{\prime}$, if $\Pi_{n}\left(y_{1}\right)=\Pi_{n}\left(y_{2}\right)$, it is obvious; if $\Pi_{n}\left(y_{1}\right) \neq \Pi_{n}\left(y_{2}\right)$, then

$$
\begin{aligned}
& \left(y_{1}-y_{2}\right)\left(f_{n}\left(s, x, y_{1}, z\right)-f_{n}\left(s, x, y_{2}, z\right)\right) \\
= & \left(\Pi_{n}\left(y_{1}\right)-\Pi_{n}\left(y_{2}\right)\right)\left(f\left(s, x, \Pi_{n}\left(y_{1}\right), z\right)-f\left(s, x, \Pi_{n}\left(y_{2}\right), z\right)\right) \frac{y_{1}-y_{2}}{\Pi_{n}\left(y_{1}\right)-\Pi_{n}\left(y_{2}\right)} \leq 0 .
\end{aligned}
$$

We then study the following BSDE with the global Lipschitz coefficient $f_{n}$ :

$$
Y_{s}^{t, x, n}=h\left(X_{T}^{t, x}\right)+\int_{s}^{T} f_{n}\left(r, X_{r}^{t, x}, Y_{r}^{t, x, n}, Z_{r}^{t, x, n}\right) d r-\int_{s}^{T}\left\langle Z_{r}^{t, x, n}, d W_{r}\right\rangle .
$$

Notice that under the conditions of Theorem 2.4 the coefficients $h$ and $f_{n}$ satisfy Conditions (H.1), $(\text { H.2 })^{\prime}$ and (H.4)'. Hence by Theorems 2.2 and 2.3 in [26], we have the following proposition:

Proposition 3.1 ([26]) Under the conditions of Theorem 2.4, for $f_{n}$ defined in (3.1), BSDE (3.2) has a unique solution $\left(Y_{s}^{t, x, n}, Z_{s}^{t, x, n}\right) \in S^{2}\left([t, T] ; L_{\rho}^{2}\left(\mathbb{R}^{d} ; \mathbb{R}^{1}\right)\right) \times M^{2}\left([t, T] ; L_{\rho}^{2}\left(\mathbb{R}^{d} ; \mathbb{R}^{d}\right)\right)$. If we define $Y_{t}^{t, x, n}=u_{n}(t, x)$, then $u_{n}(t, x)$ is the unique weak solution of the following PDE

$$
\left\{\begin{array}{l}
\frac{\partial u_{n}}{\partial t}(t, x)=-\mathscr{L} u_{n}(t, x)-f_{n}\left(t, x, u_{n}(t, x),\left(\sigma^{*} \nabla u\right)(t, x)\right), \quad 0 \leq t \leq T, \\
u_{n}(T, x)=h(x) .
\end{array}\right.
$$


Moreover,

$$
u_{n}\left(s, X_{s}^{t, x}\right)=Y_{s}^{t, x, n},\left(\sigma^{*} \nabla u_{n}\right)\left(s, X_{s}^{t, x}\right)=Z_{s}^{t, x, n} \text { for a.a. } s \in[t, T] \text {, a.a. } x \in \mathbb{R}^{d} \text { a.s. }
$$

The key is to pass the limits in (3.2) and (3.3) in some desired sense. For this we need some estimates that go beyond those in [25] and [26]. Before we derive some useful estimations to the solution of BSDEs (3.2), we give the generalized equivalence of norm principle which is an extension of equivalence of norm principle given in [14, 2, 1] to the cases when $\varphi$ and $\Psi$ are random.

Lemma 3.2 (generalized equivalence of norm principle [25]) Let $\rho$ be the weight function defined at the beginning of Section 1 and $X$ be a diffusion process defined in (2.1), where the coefficients $b \in C_{b}^{2}\left(\mathbb{R}^{d} ; \mathbb{R}^{d}\right), \sigma \in C_{b}^{3}\left(\mathbb{R}^{d} ; \mathbb{R}^{d} \times \mathbb{R}^{d}\right)$. If $s \in[t, T], \varphi: \Omega \times \mathbb{R}^{d} \rightarrow \mathbb{R}^{1}$ is independent of the $\sigma$-field $\mathscr{F}_{t, s}^{W} \triangleq \sigma\left\{W_{r}-W_{t}, t \leq r \leq s\right\}$ and $\varphi \rho^{-1} \in L^{1}\left(\Omega \times \mathbb{R}^{d}\right)$, then there exist two constants $c>0$ and $C>0$ such that

$$
c E\left[\int_{\mathbb{R}^{d}}|\varphi(x)| \rho^{-1}(x) d x\right] \leq E\left[\int_{\mathbb{R}^{d}}\left|\varphi\left(X_{s}^{t, x}\right)\right| \rho^{-1}(x) d x\right] \leq C E\left[\int_{\mathbb{R}^{d}}|\varphi(x)| \rho^{-1}(x) d x\right] .
$$

Moreover if $\Psi: \Omega \times[t, T] \times \mathbb{R}^{d} \rightarrow \mathbb{R}^{1}, \Psi(s, \cdot)$ is independent of $\mathscr{F}_{t, s}^{W}$ and $\Psi \rho^{-1} \in L^{1}\left(\Omega \times[t, T] \times \mathbb{R}^{d}\right)$, then

$$
\begin{aligned}
& c E\left[\int_{t}^{T} \int_{\mathbb{R}^{d}}|\Psi(s, x)| \rho^{-1}(x) d x d s\right] \leq E\left[\int_{t}^{T} \int_{\mathbb{R}^{d}}\left|\Psi\left(s, X_{s}^{t, x}\right)\right| \rho^{-1}(x) d x d s\right] \\
\leq & C E\left[\int_{t}^{T} \int_{\mathbb{R}^{d}}|\Psi(s, x)| \rho^{-1}(x) d x d s\right] .
\end{aligned}
$$

First we deduce a useful estimate.

Lemma 3.3 Under Conditions (H.1), (H.2), (H.3)*, (H.4) and (H.5), if $\left(Y^{t, \cdot, n}, Z^{t, \cdot, n}\right)$ is the solution of BSDE (3.2), then we have

$$
E\left[\int_{t}^{T} \sup _{n} \int_{\mathbb{R}^{d}}\left|Y_{s}^{t, x, n}\right|^{2 p} \rho^{-1}(x) d x d s\right]+\sup _{n} E\left[\int_{t}^{T} \int_{\mathbb{R}^{d}}\left|Y_{s}^{t, x, n}\right|^{2 p-2}\left|Z_{s}^{t, x, n}\right|^{2} \rho^{-1}(x) d x d s\right]<\infty .
$$

Proof. For $M, N>0$ and $m \geq 2$, define

$$
\psi_{M}(y)=y^{2} I_{\{-M \leq y<M\}}+M(2 y-M) I_{\{y \geq M\}}-M(2 y+M) I_{\{y<-M\}}
$$

and

$$
\varphi_{N, m}(y)=y^{\frac{m}{2}} I_{\{0 \leq y<N\}}+N^{\frac{m-2}{2}}\left(\frac{m}{2} y-\frac{m-2}{2} N\right) I_{\{y \geq N\}} .
$$

Applying Itô's formula to $\mathrm{e}^{K r} \varphi_{N, m}\left(\psi_{M}\left(Y_{r}^{t, x, n}\right)\right)$ for a.a. $x \in \mathbb{R}^{d}$, we have

$$
\begin{aligned}
& \mathrm{e}^{K s} \varphi_{N, m}\left(\psi_{M}\left(Y_{s}^{t, x, n}\right)\right)+K \int_{s}^{T} \mathrm{e}^{K r} \varphi_{N, m}\left(\psi_{M}\left(Y_{r}^{t, x, n}\right)\right) d r \\
& +\frac{1}{2} \int_{s}^{T} \mathrm{e}^{K r} \varphi_{N, m}^{\prime \prime}\left(\psi_{M}\left(Y_{r}^{t, x, n}\right)\right)\left|\psi_{M}^{\prime}\left(Y_{r}^{t, x, n}\right)\right|^{2}\left|Z_{r}^{t, x, n}\right|^{2} d r \\
& +\int_{s}^{T} \mathrm{e}^{K r} \varphi_{N, m}^{\prime}\left(\psi_{M}\left(Y_{r}^{t, x, n}\right)\right) I_{\left\{-M \leq Y_{r}^{t, x, n}<M\right\}}\left|Z_{r}^{t, x, n}\right|^{2} d r
\end{aligned}
$$




$$
\begin{aligned}
= & \mathrm{e}^{K T} \varphi_{N, m}\left(\psi_{M}\left(h\left(X_{T}^{t, x}\right)\right)\right)+\int_{s}^{T} \mathrm{e}^{K r} \varphi_{N, m}^{\prime}\left(\psi_{M}\left(Y_{r}^{t, x, n}\right)\right) \psi_{M}^{\prime}\left(Y_{r}^{t, x, n}\right) f_{n}\left(r, X_{r}^{t, x}, Y_{r}^{t, x, n}, Z_{r}^{t, x, n}\right) d r \\
& -\int_{s}^{T}\left\langle\mathrm{e}^{K r} \varphi_{N, m}^{\prime}\left(\psi_{M}\left(Y_{r}^{t, x, n}\right)\right) \psi_{M}^{\prime}\left(Y_{r}^{t, x, n}\right) Z_{r}^{t, x, n}, d W_{r}\right\rangle .
\end{aligned}
$$

From [25], we note first $\left(Y^{t, \cdot, n}, Z^{t, \cdot, n}\right) \in S^{2}\left([0, T] ; L_{\rho}^{2}\left(\mathbb{R}^{d} ; \mathbb{R}^{1}\right)\right) \times M^{2}\left([0, T] ; L_{\rho}^{2}\left(\mathbb{R}^{d} ; \mathbb{R}^{d}\right)\right)$. Also it is obvious that $\varphi_{N, m}^{\prime}\left(\psi_{M}\left(Y_{r}^{t, x, n}\right)\right) \psi_{M}^{\prime}\left(Y_{r}^{t, x, n}\right)$ is bounded, hence we can use the stochastic Fubini theorem and take the conditional expectation w.r.t. $\mathscr{F}_{s}$. Note that the stochastic integral has zero conditional expectation. So if we define $\frac{\psi_{M}^{\prime}(y)}{y}=2$ when $y=0$, we have

$$
\begin{aligned}
& \int_{\mathbb{R}^{d}} \mathrm{e}^{K s} \varphi_{N, m}\left(\psi_{M}\left(Y_{s}^{t, x, n}\right)\right) \rho^{-1}(x) d x+E\left[K \int_{s}^{T} \int_{\mathbb{R}^{d}} \mathrm{e}^{K r} \varphi_{N, m}\left(\psi_{M}\left(Y_{r}^{t, x, n}\right)\right) \rho^{-1}(x) d x d r \mid \mathscr{F}_{s}\right] \\
& +\frac{1}{2} E\left[\int_{s}^{T} \int_{\mathbb{R}^{d}} \mathrm{e}^{K r} \varphi_{N, m}^{\prime \prime}\left(\psi_{M}\left(Y_{r}^{t, x, n}\right)\right)\left|\psi_{M}^{\prime}\left(Y_{r}^{t, x, n}\right)\right|^{2}\left|Z_{r}^{t, x, n}\right|^{2} \rho^{-1}(x) d x d r \mid \mathscr{F}_{s}\right] \\
& +E\left[\int_{s}^{T} \int_{\mathbb{R}^{d}} \mathrm{e}^{K r} \varphi_{N, m}^{\prime}\left(\psi_{M}\left(Y_{r}^{t, x, n}\right)\right) I_{\left\{-M \leq Y_{r}^{t, x, n}<M\right\}}\left|Z_{r}^{t, x, n}\right|^{2} \rho^{-1}(x) d x d r \mid \mathscr{F}_{s}\right] \\
= & E\left[\int_{\mathbb{R}^{d}} \mathrm{e}^{K T} \varphi_{N, m}\left(\psi_{M}\left(h\left(X_{T}^{t, x}\right)\right)\right) \rho^{-1}(x) d x \mid \mathscr{F}_{s}\right] \\
& +E\left[\int_{s}^{T} \int_{\mathbb{R}^{d}} \mathrm{e}^{K r} \varphi_{N, m}^{\prime}\left(\psi_{M}\left(Y_{r}^{t, x, n}\right)\right) \psi_{M}^{\prime}\left(Y_{r}^{t, x, n}\right) f_{n}\left(r, X_{r}^{t, x}, Y_{r}^{t, x, n}, Z_{r}^{t, x, n}\right) \rho^{-1}(x) d x d r \mid \mathscr{F}_{s}\right] \\
= & E\left[\int_{\mathbb{R}^{d}} \mathrm{e}^{K T} \varphi_{N, m}\left(\psi_{M}\left(h\left(X_{T}^{t, x}\right)\right)\right) \rho^{-1}(x) d x \mid \mathscr{F}_{s}\right] \\
& +E\left[\int_{s}^{T} \int_{\mathbb{R}^{d}} \mathrm{e}^{K r} \varphi_{N, m}^{\prime}\left(\psi_{M}\left(Y_{r}^{t, x, n}\right)\right) \frac{\psi_{M}^{\prime}\left(Y_{r}^{t, x, n}\right)}{Y_{r}^{t, x, n}} Y_{r}^{t, x, n}\right. \\
& +E\left[\int_{s}^{T} \int_{\mathbb{R}^{d}} \mathrm{e}^{K r} \varphi_{N, m}^{\prime}\left(\psi_{M}\left(Y_{r}^{t, x, n}\right)\right) \psi_{M}^{\prime}\left(Y_{r}^{t, x, n}\right)\right. \\
& +E\left[\int_{s}^{T} \int_{\mathbb{R}^{d}} \mathrm{e}^{K r} \varphi_{N, m}^{\prime}\left(\psi_{M}\left(Y_{r}^{t, x, n}\right)\right) \psi_{M}^{\prime}\left(Y_{r}^{t, x, n}\right) f_{n}\left(r, X_{r}^{t, x}, 0,0\right) \rho^{-1}(x) d x d r \mid \mathscr{F}_{s}\right] \\
\leq & E\left[\int_{\mathbb{R}^{d}} \mathrm{e}^{K T} \varphi_{N, m}\left(f_{n}\left(\psi_{M}\left(h\left(X_{T}^{t, x}\right)\right)\right) \rho^{-1}(x) d x \mid \mathscr{F}_{s}\right]\right. \\
& +L E\left[\int_{s}^{t} \int_{\mathbb{R}^{d}} \mathrm{e}^{K r}\left|\varphi_{N, m}^{\prime}\left(\psi_{M}\left(Y_{r}^{t, x, n}\right)\right)\left\|\psi_{M}^{\prime}\left(Y_{r}^{t, x, n}\right)\right\| Z_{r}^{t, x, n}\right| \rho^{-1}(x) d x d r \mid \mathscr{F}_{s}\right] \\
& +E\left[\int_{s}^{T} \int_{\mathbb{R}^{d}} \mathrm{e}^{K r}\left|\varphi_{N, m}^{\prime}\left(\psi_{M}\left(Y_{r}^{t, x, n}\right)\right)\right|\left|\psi_{M}^{\prime}\left(Y_{r}^{t, x, n}\right)\right|\left|f\left(r, X_{r}^{t, x}, 0,0\right)\right| \rho^{-1}(x) d x d r \mid \mathscr{F}_{s}\right] . \\
& \left.\left.+f_{n}\left(r, X_{r}^{t, x}, 0,0\right)\right) \rho^{-1}(x) d x d r \mid \mathscr{F}_{s}\right] \\
& \left.+\mathscr{F}_{s}\right]
\end{aligned}
$$

Taking the limit as $M \rightarrow \infty$ first, then the limit as $N \rightarrow \infty$, by the monotone convergence theorem and Young inequality, we have

$$
\begin{aligned}
& \int_{\mathbb{R}^{d}} \mathrm{e}^{K s}\left|Y_{s}^{t, x, n}\right|^{m} \rho^{-1}(x) d x+K E\left[\int_{s}^{T} \int_{\mathbb{R}^{d}} \mathrm{e}^{K r}\left|Y_{r}^{t, x, n}\right|^{m} \rho^{-1}(x) d x d r \mid \mathscr{F}_{s}\right] \\
& +\frac{m(m-1)}{2} E\left[\int_{s}^{T} \int_{\mathbb{R}^{d}} \mathrm{e}^{K r}\left|Y_{r}^{t, x, n}\right|^{m-2}\left|Z_{r}^{t, x, n}\right|^{2} \rho^{-1}(x) d x d r \mid \mathscr{F}_{s}\right]
\end{aligned}
$$




$$
\begin{aligned}
& \leq E\left[\int_{\mathbb{R}^{d}} \mathrm{e}^{K T}\left|h\left(X_{T}^{t, x}\right)\right|^{m} \rho^{-1}(x) d x \mid \mathscr{F}_{s}\right] \\
& +m L E\left[\int_{s}^{T} \int_{\mathbb{R}^{d}} \mathrm{e}^{K r}\left|Y_{r}^{t, x, n}\right|^{m-2}\left|Y_{r}^{t, x, n}\right|\left|Z_{r}^{t, x, n}\right| \rho^{-1}(x) d x d r \mid \mathscr{F}_{s}\right] \\
& +m E\left[\int_{s}^{T} \int_{\mathbb{R}^{d}} \mathrm{e}^{K r}\left|Y_{r}^{t, x, n}\right|^{m-2}\left|Y_{r}^{t, x, n}\right|\left|f\left(r, X_{r}^{t, x}, 0,0\right)\right| \rho^{-1}(x) d x d r \mid \mathscr{F}_{s}\right] \\
& \leq E\left[\int_{\mathbb{R}^{d}} \mathrm{e}^{K T}\left|h\left(X_{T}^{t, x}\right)\right|^{m} \rho^{-1}(x) d x \mid \mathscr{F}_{s}\right]+m L^{2} E\left[\int_{s}^{T} \int_{\mathbb{R}^{d}} \mathrm{e}^{K r}\left|Y_{r}^{t, x, n}\right|^{m} \rho^{-1}(x) d x d r \mid \mathscr{F}_{s}\right] \\
& +\frac{m}{4} E\left[\int_{s}^{T} \int_{\mathbb{R}^{d}} \mathrm{e}^{K r}\left|Y_{r}^{t, x, n}\right|^{m-2}\left|Z_{r}^{t, x, n}\right|^{2} \rho^{-1}(x) d x d r \mid \mathscr{F}_{s}\right] \\
& +m E\left[\int_{s}^{T} \int_{\mathbb{R}^{d}} \mathrm{e}^{K r}\left|Y_{r}^{t, x, n}\right|^{m} \rho^{-1}(x) d x d r \mid \mathscr{F}_{s}\right] \\
& +\frac{m}{4} E\left[\int_{s}^{T} \int_{\mathbb{R}^{d}} \mathrm{e}^{K r}\left|Y_{r}^{t, x, n}\right|^{m-2}\left|f\left(r, X_{r}^{t, x}, 0,0\right)\right|^{2} \rho^{-1}(x) d x d r \mid \mathscr{F}_{s}\right] \\
& \leq E\left[\int_{\mathbb{R}^{d}} \mathrm{e}^{K T}\left|h\left(X_{T}^{t, x}\right)\right|^{m} \rho^{-1}(x) d x \mid \mathscr{F}_{s}\right]+m\left(L^{2}+1\right) E\left[\int_{s}^{T} \int_{\mathbb{R}^{d}} \mathrm{e}^{K r}\left|Y_{r}^{t, x, n}\right|^{m} \rho^{-1}(x) d x d r \mid \mathscr{F}_{s}\right] \\
& +\frac{m}{4} E\left[\int_{s}^{T} \int_{\mathbb{R}^{d}} \mathrm{e}^{K r}\left|Y_{r}^{t, x, n}\right|^{m-2}\left|Z_{r}^{t, x, n}\right|^{2} \rho^{-1}(x) d x d r \mid \mathscr{F}_{s}\right] \\
& +\frac{m}{4} \cdot \frac{m-2}{m} E\left[\int_{s}^{T} \int_{\mathbb{R}^{d}}\left(\mathrm{e}^{\frac{m-2}{m} K r}\left|Y_{r}^{t, x, n}\right|^{m-2}\right)^{\frac{m}{m-2}} \rho^{-1}(x) d x d r \mid \mathscr{F}_{s}\right] \\
& +\frac{m}{4} \cdot \frac{2}{m} E\left[\int_{s}^{T} \int_{\mathbb{R}^{d}}\left(\mathrm{e}^{\frac{2}{m} K r}\left|f\left(r, X_{r}^{t, x}, 0,0\right)\right|^{2}\right)^{\frac{m}{2}} \rho^{-1}(x) d x d r \mid \mathscr{F}_{s}\right] .
\end{aligned}
$$

Here and in the following, $C_{p}$ is a generic constant. Therefore, taking $K>m\left(L^{2}+1\right)+\frac{m-2}{4}$, we have

$$
\begin{aligned}
& E\left[\int_{t}^{T} \sup _{n} \int_{\mathbb{R}^{d}}\left|Y_{s}^{t, x, n}\right|^{m} \rho^{-1}(x) d x d s\right]+\sup _{n} E\left[\int_{t}^{T} \int_{\mathbb{R}^{d}}\left|Y_{s}^{t, x, n}\right|^{m-2}\left|Z_{s}^{t, x, n}\right|^{2} \rho^{-1}(x) d x d s\right] \\
\leq & C_{p} E\left[\int_{\mathbb{R}^{d}}\left|h\left(X_{T}^{t, x}\right)\right|^{m} \rho^{-1}(x) d x\right]+C_{p} E\left[\int_{t}^{T} \int_{\mathbb{R}^{d}}\left|f_{0}\left(s, X_{s}^{t, x}\right)\right|^{m} \rho^{-1}(x) d x d s\right] \\
\leq & C_{p} \int_{\mathbb{R}^{d}}|h(x)|^{m} \rho^{-1}(x) d x+C_{p} \int_{t}^{T} \int_{\mathbb{R}^{d}}\left|f_{0}(s, x)\right|^{m} \rho^{-1}(x) d x d s<\infty .
\end{aligned}
$$

In particular, taking $m=2 p$, then the lemma follows.

Taking $m=2$ in the proof of Lemma 3.3, we know

$$
E\left[\int_{t}^{T} \sup _{n} \int_{\mathbb{R}^{d}}\left|Y_{s}^{t, x, n}\right|^{2} \rho^{-1}(x) d x d s+\sup _{n} E\left[\int_{t}^{T} \int_{\mathbb{R}^{d}}\left|Z_{s}^{t, x, n}\right|^{2} \rho^{-1}(x) d x d s\right]<\infty .\right.
$$

Also we have

$$
\begin{aligned}
& \sup _{n} E\left[\int_{t}^{T} \int_{\mathbb{R}^{d}}\left|f_{n}\left(s, X_{s}^{t, x}, Y_{s}^{t, x, n}, Z_{s}^{t, x, n}\right)\right|^{2} \rho^{-1}(x) d x d s\right] \\
\leq & \sup _{n} E\left[\int_{t}^{T} \int_{\mathbb{R}^{d}} C\left(\left|f_{0}\left(s, X_{s}^{t, x}\right)\right|^{2}+\left|Y_{s}^{t, x, n}\right|^{2 p}+\left|Z_{s}^{t, x, n}\right|^{2}\right) \rho^{-1}(x) d x d s\right]<\infty .
\end{aligned}
$$


The last inequality follows from the equivalence of norms principle and Lemma 3.3. Define $U_{s}^{t, x, n}=$ $f_{n}\left(s, X_{s}^{t, x}, Y_{s}^{t, x, n}, Z_{s}^{t, x, n}\right), s \geq t$, then

$$
\sup _{n} E\left[\int_{t}^{T} \int_{\mathbb{R}^{d}}\left(\left|Y_{s}^{t, x, n}\right|^{2}+\left|Z_{s}^{t, x, n}\right|^{2}+\left|U_{s}^{t, x, n}\right|^{2}\right) \rho^{-1}(x) d x d s\right]<\infty .
$$

Therefore by using the Alaoglu lemma, we know that there exists a subsequence, still denoted by $\left(Y_{s}^{t, x, n}, Z_{s}^{t, x, n}, U_{s}^{t, x, n}\right)$, s.t. $\left(Y_{s}^{t, x, n}, Z_{s}^{t, x, n}, U_{s}^{t, x, n}\right)$ converges weakly to the limit $\left(Y_{s}^{t, x}, Z_{s}^{t, x}, U_{s}^{t, x}\right)$ in $L_{\rho}^{2}\left(\Omega \times[t, T] \times \mathbb{R}^{d} ; \mathbb{R}^{1} \times \mathbb{R}^{d} \times \mathbb{R}^{1}\right)$ (or equivalently $L^{2}\left(\Omega \times[t, T] ; L_{\rho}^{2}\left(\mathbb{R}^{d} ; \mathbb{R}^{1}\right) \times L_{\rho}^{2}\left(\mathbb{R}^{d} ; \mathbb{R}^{d}\right) \times L_{\rho}^{2}\left(\mathbb{R}^{d} ; \mathbb{R}^{1}\right)\right.$ ). Now we take the weak limit in $\left.L_{\rho}^{2}\left(\Omega \times[t, T] \times \mathbb{R}^{d} ; \mathbb{R}^{1}\right)\right)$ to BSDEs (3.2), we can verify that $\left(Y_{s}^{t, x}, Z_{s}^{t, x}, U_{s}^{t, x}\right)$ satisfies the following BSDE:

$$
Y_{s}^{t, x}=h\left(X_{T}^{t, x}\right)+\int_{s}^{T} U_{r}^{t, x} d r-\int_{s}^{T}\left\langle Z_{r}^{t, x}, d W_{r}\right\rangle .
$$

For this, we will check the weak convergence term by term. The weak convergence to the first term is deduced by the definition of $Y_{s}^{t, x}$. The weak convergence to the second term is trivial since $h\left(X_{T}^{t, x}\right)$ is independent of $n$. We then check the weak convergence to the last two terms. Let $\eta \in L_{\rho}^{2}\left(\Omega \times[t, T] \times \mathbb{R}^{d} ; \mathbb{R}^{1}\right)$. Then noticing $\int_{t}^{T} \sup _{n} E\left[\int_{s}^{T} \int_{\mathbb{R}^{d}}\left|U_{r}^{t, x, n}\right|^{2} \rho^{-1}(x) d x d r\right] d s<\infty$ due to (3.8), by Lebesgue's dominated convergence theorem, we have

$$
\begin{aligned}
& \left|E\left[\int_{t}^{T} \int_{\mathbb{R}^{d}} \int_{s}^{T}\left(U_{r}^{t, x, n}-U_{r}^{t, x}\right) d r \eta(s, x) \rho^{-1}(x) d x d s\right]\right| \\
= & \left|E\left[\int_{t}^{T} \int_{s}^{T} \int_{\mathbb{R}^{d}}\left(U_{r}^{t, x, n}-U_{r}^{t, x}\right) \eta(s, x) \rho^{-1}(x) d x d r d s\right]\right| \\
\leq & \int_{t}^{T}\left|E\left[\int_{s}^{T} \int_{\mathbb{R}^{d}}\left(U_{r}^{t, x, n}-U_{r}^{t, x}\right) \eta(s, x) \rho^{-1}(x) d x d r\right]\right| d s \longrightarrow 0, \quad \text { as } n \rightarrow \infty .
\end{aligned}
$$

On the other hand we know for fixed $s$ and $x, \eta(s, x) \in L^{2}(\Omega)$. So there exists $\varphi(s, x, r)$ s.t. $\eta(s, x)=$ $E[\eta(s, x)]+\int_{t}^{T}\left\langle\varphi(s, x, r), d W_{r}\right\rangle$. It is easy to see that for a.a. $s \in[t, T], \varphi(s, \cdot, \cdot) \in L^{2}(\Omega \times[t, T] \times$ $\left.\mathbb{R}^{d} ; \mathbb{R}^{1}\right)$. Noticing that $\int_{t}^{T} \sup _{n} E\left[\int_{s}^{T} \int_{\mathbb{R}^{d}}\left|Z_{r}^{t, x, n}\right|^{2} \rho^{-1}(x) d x d r\right] d s<\infty$ due to (3.8) and using Lebesgue's dominated convergence theorem again, we obtain

$$
\begin{aligned}
& \left|E\left[\int_{t}^{T} \int_{\mathbb{R}^{d}} \int_{s}^{T}\left\langle Z_{r}^{t, x, n}-Z_{r}^{t, x}, d W_{r}\right\rangle \eta(s, x) \rho^{-1}(x) d x d s\right]\right| \\
= & \left|\int_{t}^{T} \int_{\mathbb{R}^{d}} E\left[\int_{s}^{T}\left\langle Z_{r}^{t, x, n}-Z_{r}^{t, x}, d W_{r}\right\rangle\left(E[\eta(s, x)]+\int_{t}^{T}\left\langle\varphi(s, x, r), d W_{r}\right\rangle\right)\right] \rho^{-1}(x) d x d s\right| \\
= & \left|\int_{t}^{T} \int_{\mathbb{R}^{d}} E\left[\int_{s}^{T}\left\langle Z_{r}^{t, x, n}-Z_{r}^{t, x}, \varphi(s, x, r)\right\rangle d r\right] \rho^{-1}(x) d x d s\right| \\
\leq & \int_{t}^{T}\left|E\left[\int_{s}^{T} \int_{\mathbb{R}^{d}}\left\langle Z_{r}^{t, x, n}-Z_{r}^{t, x}, \varphi(s, x, r)\right\rangle \rho^{-1}(x) d x d r\right]\right| d s \longrightarrow 0, \quad \text { as } n \rightarrow \infty .
\end{aligned}
$$

Needless to say, if we can show BSDE (3.2) is indeed BSDE (2.2), then we can say $\left(Y_{s}^{t, x}, Z_{s}^{t, x}\right)$ is a solution of BSDE (2.2). The key is to prove that $U_{s}^{t, x}=f\left(s, X_{s}^{t, x}, Y_{s}^{t, x}, Z_{s}^{t, x}\right)$ for a.a. $s \in[t, T], x \in \mathbb{R}^{d}$ a.s. However, the weak convergence of $Y^{n}, U^{n}$ and $Z^{n}$ are not enough to this. The crucial point in this analysis is to establish the strong convergence of $Y^{n}$ and $Z^{n}$, which will be done in next section. 


\section{The strong convergence and the identification of the limiting BSDEs}

In this section, we will show that the combination of methods of weak convergence and strong convergence of a subsequence $\left(Y_{s}^{t, x, n}, Z_{s}^{t, x, n}\right)$ gives an effective way to prove that the limit $\left(Y_{s}^{t, x}, Z_{s}^{t, x}\right)$ satisfies BSDE (2.2). In contrast, the direct proof that BSDE (3.2) converges strongly to BSDE (2.2) by using the strongly convergent subsequence $\left(Y_{s}^{t, x, n}, Z_{s}^{t, x, n}\right)$ without the weak convergence argument will encounter some complications. This is due to that the dominated convergence theorem does not seem to apply immediately to BSDE (3.2). We start from an easy lemma.

Lemma 4.1 Under the conditions of Theorem 2.4, if $u_{n}(t, x)$ is the weak solution of PDE (3.3), then $\sup _{n} \int_{0}^{T} \int_{\mathbb{R}^{d}}\left|u_{n}(s, x)\right|^{2 p} \rho^{-1}(x) d x d s<\infty$. Furthermore,

$$
\lim _{N \rightarrow \infty} \sup _{n} \int_{0}^{T} \int_{\mathbb{R}^{d}}\left|u_{n}(s, x)\right|^{2} I_{U_{N}}{ }(x) \rho^{-1}(x) d x d s=0,
$$

where $U_{N}^{c}=\left\{x \in \mathbb{R}^{d}:|x|>N\right\}$.

Proof. By the equivalence of norm principle, (3.4) and Lemma 3.3, we deduce the $L_{\rho}^{2 p}$ integrability of $u_{n}$ as follows:

$$
\begin{aligned}
\sup _{n} \int_{0}^{T} \int_{\mathbb{R}^{d}}\left|u_{n}(s, x)\right|^{2 p} \rho^{-1}(x) d x d s & \leq C_{p} \sup _{n} E\left[\int_{0}^{T} \int_{\mathbb{R}^{d}}\left|u_{n}\left(s, X_{s}^{0, x}\right)\right|^{2 p} \rho^{-1}(x) d x d s\right] \\
& =C_{p} \sup _{n} E\left[\int_{0}^{T} \int_{\mathbb{R}^{d}}\left|Y_{s}^{0, x, n}\right|^{2 p} \rho^{-1}(x) d x d s\right]<\infty .
\end{aligned}
$$

Let's then prove the second part of this lemma. Since $\int_{\mathbb{R}^{d}} \rho^{-1}(x) d x<\infty$,

$$
\begin{aligned}
& \lim _{N \rightarrow \infty} \sup _{n} \int_{0}^{T} \int_{\mathbb{R}^{d}}\left|u_{n}(s, x)\right|^{2} I_{U_{N}}(x) \rho^{-1}(x) d x d s \\
\leq & \lim _{N \rightarrow \infty}\left(\sup _{n} \int_{0}^{T} \int_{\mathbb{R}^{d}}\left|u_{n}(s, x)\right|^{2 p} \rho^{-1}(x) d x d s\right)^{\frac{1}{p}}\left(\int_{0}^{T} \int_{\mathbb{R}^{d}}\left|I_{U_{N}}(x)\right|^{\frac{p}{p-1}} \rho^{-1}(x) d x d s\right)^{\frac{p-1}{p}} \\
\leq & \lim _{N \rightarrow \infty} C_{p}\left(\int_{\mathbb{R}^{d}} I_{U_{N}}(x) \rho^{-1}(x) d x\right)^{\frac{p-1}{p}}=0 .
\end{aligned}
$$

The following two theorems quoted in 21] will be used in this section.

Theorem 4.2 (c.f. [21]) Let $X \subset \subset H \subset Y$ be Banach spaces, with $X$ reflexive. Here $X \subset \subset H$ means $X$ is compactly embedded in $H$. Suppose that $u_{n}$ is a sequence that is uniformly bounded in $L^{2}([0, T] ; X)$, and $d u_{n} / d t$ is uniformly bounded in $L^{p}(0, T ; Y)$, for some $p>1$. Then there is a subsequence that converges strongly in $L^{2}([0, T] ; H)$.

Theorem 4.3 (Rellich-Kondrachov Compactness Theorem c.f. [21]) Let B be a bounded $C^{1}$ domain in $\mathbb{R}^{d}$. Then $H^{1}(B)$ is compactly embedded in $L^{2}(B)$.

Lemma 4.4 Under the conditions of Theorem 2.4. if $\left(Y_{s}^{t, x, n}, Z_{s}^{t, x, n}\right)$ is the solution of BSDEs (3.2) and $Y_{s}^{t, x}$ is the weak limit of $Y_{s}^{t, x, n}$ in $L_{\rho}^{2}\left(\Omega \times[t, T] \times \mathbb{R}^{d} ; \mathbb{R}^{1}\right)$, then there is a subsequence of $Y_{s}^{t, x, n}$, still denoted by $Y_{s}^{t, x, n}$, converging strongly to $Y_{s}^{t, x}$ in $L^{2}\left(\Omega \times[t, T] ; L_{\rho}^{2}\left(\mathbb{R}^{d} ; \mathbb{R}^{1}\right)\right)$. 
Proof. Let $u_{n}(s, x)=Y_{s}^{s, x, n}$. Then by Proposition 3.1 $u_{n}\left(s, X_{s}^{t, x}\right)=Y_{s}^{t, x, n},\left(\sigma^{*} \nabla u_{n}\right)\left(s, X_{s}^{t, x}\right)=Z_{s}^{t, x, n}$ for a.a. $s \in[t, T], x \in \mathbb{R}^{d}$ a.s. Moreover, $u_{n}(s, x)$ is a weak solution of the PDE (3.3). By the definition of weak solution and the fact that $C_{c}^{\infty}\left(\mathbb{R}^{d} ; \mathbb{R}^{1}\right)$ is dense in $H_{\rho}^{1}\left(\mathbb{R}^{d} ; \mathbb{R}^{1}\right), u_{n}(s, x)$ satisfies the following $\mathrm{PDE}$ in $H_{\rho}^{1^{*}}\left(\mathbb{R}^{d} ; \mathbb{R}^{1}\right)$ :

$$
d u_{n}(s, x) / d s=-\mathscr{L} u_{n}(s, x)-f_{n}\left(s, x, u_{n}(s, x),\left(\sigma^{*} \nabla u_{n}\right)(s, x)\right), \quad 0 \leq s \leq T .
$$

To get a strongly convergent subsequence of $Y_{s}^{t, x, n}$, first note that $u_{n}$ are uniformly bounded in $L^{2}\left([0, T] ; H_{\rho}^{1}\left(\mathbb{R}^{d} ; \mathbb{R}^{1}\right)\right)$ by the uniform ellipticity condition of $\sigma$ and the equivalence of norm principle:

$$
\begin{aligned}
& \sup _{n} \int_{0}^{T} \int_{\mathbb{R}^{d}}\left(\left|u_{n}(s, x)\right|^{2}+\left|\nabla u_{n}(s, x)\right|^{2}\right) \rho^{-1}(x) d x d s \\
\leq & C_{p} \sup _{n} \int_{0}^{T} \int_{\mathbb{R}^{d}}\left(\left|u_{n}(s, x)\right|^{2}+\left|\left(\sigma^{*} \nabla u_{n}\right)(s, x)\right|^{2}\right) \rho^{-1}(x) d x d s \\
\leq & C_{p} \sup _{n} E\left[\int_{0}^{T} \int_{\mathbb{R}^{d}}\left(\left|Y_{s}^{0, x, n}\right|^{2}+\left|Z_{s}^{0, x, n}\right|^{2}\right) \rho^{-1}(x) d x d s\right]<\infty .
\end{aligned}
$$

Then we can deduce that $d u_{n} / d s$ are uniformly bounded in $L^{2}\left([0, T] ; H_{\rho}^{1^{*}}\left(\mathbb{R}^{d} ; \mathbb{R}^{1}\right)\right)$. For this, we need to prove that $\mathscr{L} u_{n}$ and $f_{n} \in L^{2}\left([0, T] ; H_{\rho}^{1^{*}}\left(\mathbb{R}^{d} ; \mathbb{R}^{1}\right)\right)$ are uniformly bounded respectively. First note that for $i=1,2, \cdots, d$,

$$
\left|\frac{\partial \rho^{-1}(x)}{\partial x_{i}}\right|=\left|\frac{-q x_{i}}{(1+|x|)^{q+1}|x|}\right| \leq \frac{q}{(1+|x|)^{q+1}} \leq q \rho^{-1}(x) .
$$

Moreover, recalling the form of $\mathscr{L}$ and noticing the conditions on $b$ and $\sigma$ in (H.5), we can see that $a_{i j}$ and $b_{i}$ are uniformly bounded for all $i, j$. So for arbitrary $s \in[0, T], \psi \in C_{c}^{\infty}\left(\mathbb{R}^{d} ; \mathbb{R}^{1}\right)$, we have

$$
\begin{aligned}
& \int_{\mathbb{R}^{d}} \mathscr{L} u_{n}(s, x) \cdot \psi(x) \rho^{-1}(x) d x \\
= & \int_{\mathbb{R}^{d}}\left(-\frac{1}{2} \sum_{i, j=1}^{d} \frac{\partial u_{n}(s, x)}{\partial x_{i}} \frac{\partial\left(a_{i j} \psi \rho^{-1}\right)(x)}{\partial x_{j}}-\sum_{i=1}^{d} u_{n}(s, x) \frac{\partial\left(b_{i} \psi \rho^{-1}\right)(x)}{\partial x_{i}}\right) d x \\
\leq & \int_{\mathbb{R}^{d}}\left(\sum_{i=1}^{d}\left|\frac{\partial u_{n}(s, x)}{\partial x_{i}}\right|+\left|u_{n}(s, x)\right|\right)\left(\sum_{i, j=1}^{d}\left|\frac{\partial\left(a_{i j} \psi \rho^{-1}\right)(x)}{\partial x_{j}}\right|+\sum_{i=1}^{d}\left|\frac{\partial\left(b_{i} \psi \rho^{-1}\right)(x)}{\partial x_{i}}\right|\right) d x \\
\leq & C_{p} \int_{\mathbb{R}^{d}}\left(\sum_{i=1}^{d}\left|\frac{\partial u_{n}(s, x)}{\partial x_{i}}\right|+\left|u_{n}(s, x)\right|\right)\left(\sum_{j=1}^{d}\left|\frac{\partial \psi(x)}{\partial x_{j}}\right|+|\psi(x)|\right) \rho^{-1}(x) d x \\
\leq & C_{p} \sqrt{\int_{\mathbb{R}^{d}}\left(\sum_{i=1}^{d}\left|\frac{\partial u_{n}(s, x)}{\partial x_{i}}\right|+\left|u_{n}(s, x)\right|\right)^{2} \rho^{-1}(x) d x} \sqrt{\int_{\mathbb{R}^{d}}\left(\sum_{j=1}^{d}\left|\frac{\partial \psi(x)}{\partial x_{j}}\right|+|\psi(x)|\right)^{2} \rho^{-1}(x) d x} \\
\leq & C_{p}\left\|u_{n}(s, x)\right\|_{H_{\rho}^{1}\left(\mathbb{R}^{d} ; \mathbb{R}^{1}\right)}\|\psi\|_{H_{\rho}^{1}\left(\mathbb{R}^{d} ; \mathbb{R}^{1}\right) .}
\end{aligned}
$$

As $C_{c}^{\infty}\left(\mathbb{R}^{d} ; \mathbb{R}^{1}\right)$ is dense in $H_{\rho}^{1}\left(\mathbb{R}^{d} ; \mathbb{R}^{1}\right)$, therefore for arbitrary $s \in[0, T]$, it follows that $\left\|\mathscr{L} u_{n}(s, \cdot)\right\|_{H_{\rho}^{1 *}\left(\mathbb{R}^{d} ; \mathbb{R}^{1}\right)}$ $\leq C_{p}\left\|u_{n}(s, \cdot)\right\|_{H_{\rho}^{1}\left(\mathbb{R}^{d} ; \mathbb{R}^{1}\right)}$ and by (4.2), we have

$$
\sup _{n}\left\|\mathscr{L} u_{n}\right\|_{L^{2}\left([0, T] ; H_{\rho}^{1 *}\left(\mathbb{R}^{d} ; \mathbb{R}^{1}\right)\right)}^{2} \leq C_{p} \sup _{n} \int_{0}^{T} \int_{\mathbb{R}^{d}}\left(\left|u_{n}(s, x)\right|^{2}+\left|\nabla u_{n}(s, x)\right|^{2}\right) \rho^{-1}(x) d x d s<\infty .
$$


Also using Lemma 3.3 and the equivalence of norm principle again, we obtain

$$
\begin{aligned}
& \int_{0}^{T}\left\|f_{n}\left(s, \cdot, u_{n}(s, \cdot),\left(\sigma^{*} \nabla u_{n}\right)(s, \cdot)\right)\right\|_{L_{\rho}^{2}\left(\mathbb{R}^{d} ; \mathbb{R}^{1}\right)}^{2} d s \\
\leq & C_{p} E\left[\int_{0}^{T} \int_{\mathbb{R}^{d}}\left(\left|f_{0}(s, x)\right|^{2}+\left|Y_{s}^{0, x, n}\right|^{2 p}+\left|Z_{s}^{0, x, n}\right|^{2}\right) \rho^{-1}(x) d x d s\right]<\infty .
\end{aligned}
$$

Hence $f_{n} \in L^{2}\left([0, T] ; L_{\rho}^{2^{*}}\left(\mathbb{R}^{d} ; \mathbb{R}^{1}\right)\right) \subset L^{2}\left([0, T] ; H_{\rho}^{1^{*}}\left(\mathbb{R}^{d} ; \mathbb{R}^{1}\right)\right)$ and

$$
\begin{aligned}
& \sup _{n}\left\|f_{n}\right\|_{L^{2}\left([0, T] ; H_{\rho}^{1 *}\left(\mathbb{R}^{d} ; \mathbb{R}^{1}\right)\right)}^{2} \\
\leq & C_{p} \sup _{n} \int_{0}^{T}\left\|f_{n}\left(s, \cdot, u_{n}(s, \cdot),\left(\sigma^{*} \nabla u_{n}\right)(s, \cdot)\right)\right\|_{L_{\rho}^{2}\left(\mathbb{R}^{d} ; \mathbb{R}^{1}\right)}^{2} d s \\
\leq & C_{p} \sup _{n} E\left[\int_{0}^{T} \int_{\mathbb{R}^{d}}\left(\left|f_{0}(s, x)\right|^{2}+\left|Y_{s}^{0, x, n}\right|^{2 p}+\left|Z_{s}^{0, x, n}\right|^{2}\right) \rho^{-1}(x) d x d s\right]<\infty .
\end{aligned}
$$

Therefore we conclude that $d u_{n} / d s$ are uniformly bounded in $L^{2}\left([0, T] ; H_{\rho}^{1^{*}}\left(\mathbb{R}^{d} ; \mathbb{R}^{1}\right)\right)$.

Noticing Theorem 4.3 and applying Theorem 4.2 with $X=H_{\rho}^{1}\left(U_{1} ; \mathbb{R}^{1}\right), H=L_{\rho}^{2}\left(U_{1} ; \mathbb{R}^{1}\right)$ and $Y=H_{\rho}^{1^{*}}\left(U_{1} ; \mathbb{R}^{1}\right)$, we are able to extract a subsequence of $u_{n}(s, x)$, denoted by $u_{1 n}(s, x)$, which converges strongly in $L^{2}\left([0, T] ; L_{\rho}^{2}\left(U_{1} ; \mathbb{R}^{1}\right)\right)$. It is obvious that this $u_{1 n}(s, x)$ satisfies the conditions in Theorem 4.2. Applying Theorem 4.2 again, we are able to extract a subsequence of $u_{1 n}(s, x)$, denoted by $u_{2 n}(s, x)$, that converges strongly in $L^{2}\left([0, T] ; L_{\rho}^{2}\left(U_{2} ; \mathbb{R}^{1}\right)\right)$. Actually we can do this procedure for all $U_{i}, i=1,2, \cdots$. Now we pick up the diagonal sequence $u_{i i}(s, x), i=1,2, \cdots$ and still denote this sequence by $u_{n}$ for convenience. It is easy to see that $u_{n}$ converges strongly in all $L^{2}\left([0, T] ; L_{\rho}^{2}\left(U_{i} ; \mathbb{R}^{1}\right)\right)$, $i=1,2, \cdots$. For arbitrary $\varepsilon>0$, noticing Lemma 4.1, we can find $j(\varepsilon)$ large enough such that

$$
\sup _{n} \int_{0}^{T} \int_{U_{j(\varepsilon)}} 2\left|u_{n}(s, x)\right|^{2} \rho^{-1}(x) d x d s<\frac{\varepsilon}{3} .
$$

For this $j(\varepsilon)$, there exists $n^{*}(\varepsilon)>0$ s.t. when $m, n \geq n^{*}(\varepsilon)$, we know

$$
\left\|u_{m}-u_{n}\right\|_{L^{2}\left([0, T] ; L_{\rho}^{2}\left(U_{j(\varepsilon)} ; \mathbb{R}^{1}\right)\right)}^{2}=\int_{0}^{T} \int_{U_{j(\varepsilon)}}\left|u_{m}(s, x)-u_{n}(s, x)\right|^{2} \rho^{-1}(x) d x d s<\frac{\varepsilon}{3} .
$$

Therefore as $m, n \geq n^{*}(\varepsilon)$,

$$
\begin{aligned}
& \left\|u_{m}-u_{n}\right\|_{L^{2}\left([0, T] ; L_{\rho}^{2}\left(\mathbb{R}^{d} ; \mathbb{R}^{1}\right)\right)}^{2} \\
\leq & \int_{0}^{T} \int_{U_{j(\varepsilon)}}\left|u_{m}(s, x)-u_{n}(s, x)\right|^{2} \rho^{-1}(x) d x d s+\int_{0}^{T} \int_{U_{j(\varepsilon)}{ }^{c}}\left(2\left|u_{m}(s, x)\right|^{2}+2\left|u_{n}(s, x)\right|^{2}\right) \rho^{-1}(x) d x d s \\
< & \varepsilon
\end{aligned}
$$

That is to say $u_{n}$ converges strongly in $L^{2}\left([0, T] ; L_{\rho}^{2}\left(\mathbb{R}^{d} ; \mathbb{R}^{1}\right)\right)$. Now using the equivalence of norm principle, we know as $m, n \rightarrow \infty$,

$$
\begin{aligned}
& \left\|Y_{s}^{t, x, m}-Y_{s}^{t, x, n}\right\|_{L^{2}\left(\Omega \times[t, T] ; L_{\rho}^{2}\left(\mathbb{R}^{d} ; \mathbb{R}^{1}\right)\right)}^{2} \\
= & E\left[\int_{t}^{T} \int_{\mathbb{R}^{d}}\left|u_{m}\left(s, X_{s}^{t, x}\right)-u_{n}\left(s, X_{s}^{t, x}\right)\right|^{2} \rho^{-1}(x) d x d s\right]
\end{aligned}
$$




$$
\leq C_{p} \int_{t}^{T} \int_{\mathbb{R}^{d}}\left|u_{m}(s, x)-u_{n}(s, x)\right|^{2} \rho^{-1}(x) d x d s \longrightarrow 0 .
$$

So the claim that $Y_{s}^{t, x, n}$ converges strongly in $L^{2}\left(\Omega \times[t, T] ; L_{\rho}^{2}\left(\mathbb{R}^{d} ; \mathbb{R}^{1}\right)\right)$ follows. But we know that $Y_{s}^{t, x}$ is the weak limit of $Y_{s}^{t, x, n}$ in $L^{2}\left(\Omega \times[t, T] ; L_{\rho}^{2}\left(\mathbb{R}^{d} ; \mathbb{R}^{1}\right)\right)$, therefore $Y_{s}^{t, x, n}$ converges strongly to $Y_{s}^{t, x}$ in $L^{2}\left(\Omega \times[t, T] ; L_{\rho}^{2}\left(\mathbb{R}^{d} ; \mathbb{R}^{1}\right)\right)$.

Considering the strongly convergent subsequence $\left\{Y^{t, \cdot, n}\right\}_{n=1}^{\infty}$ derived from Lemma 4.4 and using a standard argument to BSDE (3.2), we can prove that for arbitrary $m, n$

$$
\begin{aligned}
& E\left[\sup _{t \leq s \leq T} \int_{\mathbb{R}^{d}}\left|Y_{s}^{t, x, m}-Y_{s}^{t, x, n}\right|^{2} \rho^{-1}(x) d x\right]+E\left[\int_{t}^{T} \int_{\mathbb{R}^{d}}\left|Z_{s}^{t, x, m}-Z_{s}^{t, x, n}\right|^{2} \rho^{-1}(x) d x d s\right] \\
\leq & C_{p} E\left[\int_{t}^{T} \int_{\mathbb{R}^{d}}\left|Y_{s}^{t, x, m}-Y_{s}^{t, x, n}\right|^{2} \rho^{-1}(x) d x d s\right]+C_{p} \sqrt{E\left[\int_{t}^{T} \int_{\mathbb{R}^{d}}\left|Y_{s}^{t, x, m}-Y_{s}^{t, x, n}\right|^{2} \rho^{-1}(x) d x d s\right]} \\
& \times \sqrt{E\left[\int_{t}^{T} \int_{\mathbb{R}^{d}}\left(\left|f_{0}(s, x)\right|^{2}+\left|Y_{s}^{t, x, n}\right|^{2 p}+\left|Z_{s}^{t, x, n}\right|^{2}\right) \rho^{-1}(x) d x d s\right] .}
\end{aligned}
$$

So by Condition (H.2) and Lemma 3.3, we can conclude that this subsequence $\left\{Y^{t, \cdot, n}\right\}_{n=1}^{\infty}$ converges strongly also in $S^{2}\left([t, T] ; L_{\rho}^{2}\left(\mathbb{R}^{d} ; \mathbb{R}^{1}\right)\right)$ and the corresponding subsequence of $\left\{Z^{t, \cdot, n}\right\}_{n=1}^{\infty}$ converges strongly $M^{2}\left([t, T] ; L_{\rho}^{2}\left(\mathbb{R}^{d} ; \mathbb{R}^{d}\right)\right)$ as well. Certainly the strong convergence limit should be identified with the weak convergence limit $Z^{t, \cdot}$, hence the following corollary follows without a surprise.

Corollary 4.5 Let $\left(Y^{t, \cdot}, Z^{t, \cdot}\right)$ be the solution to BSDE (3.9) and $\left(Y^{t, \cdot, n}, Z^{t, \cdot, n}\right)$ be the subsequence of the solutions to BSDE (3.2), of which $Y^{t, \cdot, n}$ converges strongly to $Y^{t, \cdot}$ in $L^{2}\left(\Omega \times[t, T] ; L_{\rho}^{2}\left(\mathbb{R}^{d} ; \mathbb{R}^{1}\right)\right)$, then $\left(Y^{t, \cdot, n}, Z^{t, \cdot, n}\right)$ also converges strongly to $\left(Y^{t, \cdot}, Z^{t, \cdot}\right)$ in $S^{2}\left([t, T] ; L_{\rho}^{2}\left(\mathbb{R}^{d} ; \mathbb{R}^{1}\right)\right) \times M^{2}\left([t, T] ; L_{\rho}^{2}\left(\mathbb{R}^{d} ; \mathbb{R}^{d}\right)\right)$.

As for $Y_{s}^{t, x}$, we further have

Lemma 4.6 Under the conditions of Theorem 2.4. $E\left[\int_{t}^{T} \int_{\mathbb{R}^{d}}\left|Y_{s}^{t, x}\right|^{2 p} \rho^{-1}(x) d x d s\right]<\infty$ and $Y_{s}^{t, x}=$ $Y_{s}^{s, X_{s}^{t, x}}$ for a.a. $s \in[t, T]$, a.a. $x \in \mathbb{R}^{d}$ a.s.

Proof. First by Lemma 3.2 and Corollary 4.5, we have

$$
\begin{aligned}
& E\left[\int_{t}^{T} \int_{\mathbb{R}^{d}}\left|Y_{s}^{t, x}-Y_{s}^{s, X_{s}^{t, x}}\right|^{2} \rho^{-1}(x) d x d s\right] \\
\leq & \lim _{n \rightarrow \infty} 2 E\left[\int_{t}^{T} \int_{\mathbb{R}^{d}}\left|Y_{s}^{t, x, n}-Y_{s}^{t, x}\right|^{2} \rho^{-1}(x) d x d s\right] \\
& +\lim _{n \rightarrow \infty} 2 E\left[\int_{t}^{T} \int_{\mathbb{R}^{d}}\left|Y_{s}^{s, X_{s}^{t, x}, n}-Y_{s}^{s, X_{s}^{t, x}}\right|^{2} \rho^{-1}(x) d x d s\right] \\
\leq & \lim _{n \rightarrow \infty} 2 E\left[\int_{t}^{T} \int_{\mathbb{R}^{d}}\left|Y_{s}^{t, x, n}-Y_{s}^{t, x}\right|^{2} \rho^{-1}(x) d x d s\right] \\
& +\lim _{n \rightarrow \infty} C_{p} E\left[\sup _{s \leq r \leq T} \int_{\mathbb{R}^{d}}\left|Y_{r}^{s, x, n}-Y_{r}^{s, x}\right|^{2} \rho^{-1}(x) d x\right]=0 .
\end{aligned}
$$

Hence,

$$
Y_{s}^{t, x}=Y_{s}^{s, X_{s}^{t, x}} \text { for a.a. } s \in[t, T] \text {, a.a. } x \in \mathbb{R}^{d} \text { a.s. }
$$


If we define $Y_{s}^{s, x}=u(s, x)$, then by (4.4) and Lemma 3.2 again we also have

$$
\lim _{n \rightarrow \infty} \int_{0}^{T} \int_{\mathbb{R}^{d}}\left|u_{n}(s, x)-u(s, x)\right|^{2} \rho^{-1}(x) d x d s=0,
$$

and

$$
E\left[\int_{t}^{T} \int_{\mathbb{R}^{d}}\left|Y_{s}^{t, x}-u\left(s, X_{s}^{t, x}\right)\right|^{2} \rho^{-1}(x) d x d s\right]=0 .
$$

Therefore, we claim that the strong limit of $u_{n}(s, x)$ in $L^{2}\left([0, T] ; L_{\rho}^{2}\left(\mathbb{R}^{d} ; \mathbb{R}^{1}\right)\right)$ is $u(s, x)$ and $Y_{s}^{t, x}=$ $u\left(s, X_{s}^{t, x}\right)$ for a.a. $s \in[t, T], x \in \mathbb{R}^{d}$ a.s.

By the equivalence of norm principle, to get $E\left[\int_{t}^{T} \int_{\mathbb{R}^{d}}\left|Y_{s}^{t, x}\right|^{2 p} \rho^{-1}(x) d x d s\right]<\infty$, we only need to prove $\int_{0}^{T} \int_{\mathbb{R}^{d}}|u(s, x)|^{2 p} \rho^{-1}(x) d x d s<\infty$. For this, we first derive from $\lim _{n \rightarrow \infty} \int_{0}^{T} \int_{\mathbb{R}^{d}} \mid u_{n}(s, x)-$ $\left.u(s, x)\right|^{2} \rho^{-1}(x) d x d s=0$ a subsequence of $\left\{u_{n}(s, x)\right\}_{n=1}^{\infty}$, still denoted by $\left\{u_{n}(s, x)\right\}_{n=1}^{\infty}$, s.t.

$$
u_{n}(s, x) \longrightarrow u(s, x) \text { and } \sup _{n}\left|u_{n}(s, x)\right|^{2 p}<\infty \text { for a.a. } s \in[t, T], x \in \mathbb{R}^{d} .
$$

By a similar argument as in Lemma 4.1, for this subsequence $u_{n}$, we can prove that for any $\delta>0$,

$$
\lim _{N \rightarrow \infty} \sup _{n} \int_{0}^{T} \int_{\mathbb{R}^{d}}\left|u_{n}(s, x)\right|^{2 p-\delta} I_{\left\{\left|u_{n}(s, x)\right|^{2 p-\delta}>N\right\}}(s, x) \rho^{-1}(x) d x d s=0 .
$$

That is to say that $\left|u_{n}(s, x)\right|^{2 p-\delta}$ is uniformly integrable. Together with $u_{n}(s, x) \longrightarrow u(s, x)$ for a.a. $s \in[0, T], x \in \mathbb{R}^{d}$, we have

$$
\begin{aligned}
& \int_{0}^{T} \int_{\mathbb{R}^{d}}|u(s, x)|^{2 p-\delta} \rho^{-1}(x) d x d s=\lim _{n \rightarrow \infty} \int_{0}^{T} \int_{\mathbb{R}^{d}}\left|u_{n}(s, x)\right|^{2 p-\delta} \rho^{-1}(x) d x d s \\
\leq & \sup _{n} \int_{0}^{T} \int_{\mathbb{R}^{d}}\left|u_{n}(s, x)\right|^{2 p-\delta} \rho^{-1}(x) d x d s \leq C_{p}\left(\sup _{n} \int_{0}^{T} \int_{\mathbb{R}^{d}}\left|u_{n}(s, x)\right|^{2 p} \rho^{-1}(x) d x d s\right)^{\frac{2 p-\delta}{2 p}} \leq C_{p},
\end{aligned}
$$

where the last $C_{p}<\infty$ is a constant independent of $n$ and $\delta$. Then using Fatou lemma to take the limit as $\delta \rightarrow 0$ in the above inequality, we can get $\int_{0}^{T} \int_{\mathbb{R}^{d}}|u(s, x)|^{2 p} \rho^{-1}(x) d x d s<\infty$.

Indeed, with Corollary 4.5 and Lemma4.6 doing Itô's formula to $\psi_{M}\left(Y_{r}^{t, x, n}-Y_{r}^{t, x}\right)$ and $\mathrm{e}^{K r} \varphi_{n, m}\left(\psi_{M}\left(Y_{r}^{t, x}\right)\right)$, we can further prove that $Y^{t, \cdot} \in S^{2 p}\left([t, T] ; L_{\rho}^{2 p}\left(\mathbb{R}^{d} ; \mathbb{R}^{1}\right)\right.$ ) (To see similar calculations, one can refer to the argument in the proof of Lemma 3.3 in [25]).


$\left.\left(\mathbb{R}^{d} ; \mathbb{R}^{1}\right)\right)$.

Now we are ready to prove the identification of the limiting BSDEs.

Lemma 4.8 The random field $U, Y$ and $Z$ have the following relation:

$$
U_{s}^{t, x}=f\left(s, X_{s}^{t, x}, Y_{s}^{t, x}, Z_{s}^{t, x}\right) \text { for a.a. } \mathrm{s} \in[\mathrm{t}, \mathrm{T}], \mathrm{x} \in \mathbb{R}^{\mathrm{d}} \text { a.s. }
$$

Proof. Let $\mathcal{K}$ be a set in $\Omega \times[t, T] \times \mathbb{R}^{d}$ s.t. $\sup _{n}\left|Y_{s}^{t, x, n}\right|+\sup _{n}\left|Z_{s}^{t, x, n}\right|+\left|f_{0}\left(s, X_{s}^{t, x}\right)\right|<K$. Similar to (4.6), we can find a subsequence of $\left\{\left(Y_{s}^{t, x, n}, Z_{s}^{t, x, n}\right)\right\}_{n=1}^{\infty}$, still denoted by $\left\{\left(Y_{s}^{t, x, n}, Z_{s}^{t, x, n}\right)\right\}_{n=1}^{\infty}$, satisfying $\left(Y_{s}^{t, x, n}, Z_{s}^{t, x, n}\right) \longrightarrow\left(Y_{s}^{t, x}, Z_{s}^{t, x}\right)$ and $\sup _{n}\left|Y_{s}^{t, x, n}\right|+\sup _{n}\left|Z_{s}^{t, x, n}\right|<\infty$ for a.a. $s \in[t, T]$, 
$x \in \mathbb{R}^{d}$ a.s. Then it turns out that as $K \rightarrow \infty, \mathcal{K} \uparrow \Omega \times[t, T] \times \mathbb{R}^{d}$. Moreover it is easy to see that along the subsequence,

$$
\begin{aligned}
& E\left[\int_{t}^{T} \int_{\mathbb{R}^{d}} 2\left(\sup _{n}\left|f_{n}\left(s, X_{s}^{t, x}, Y_{s}^{t, x, n}, Z_{s}^{t, x, n}\right)\right|^{2}+\left|f\left(s, X_{s}^{t, x}, Y_{s}^{t, x}, Z_{s}^{t, x}\right)\right|^{2}\right) I_{\mathcal{K}}(s, x) \rho^{-1}(x) d x d s\right] \\
\leq & 6 C^{2} E\left[\int_{t}^{T} \int_{\mathbb{R}^{d}}\left(\left|f_{0}\left(s, X_{s}^{t, x}\right)\right|^{2}+\sup _{n}\left|Y_{s}^{t, x, n}\right|^{2 p}+\sup _{n}\left|Z_{s}^{t, x, n}\right|^{2}\right) I_{\mathcal{K}}(s, x) \rho^{-1}(x) d x d s\right] \\
& +6 C^{2} E\left[\int_{t}^{T} \int_{\mathbb{R}^{d}}\left(\left|f_{0}\left(s, X_{s}^{t, x}\right)\right|^{2}+\left|Y_{s}^{t, x}\right|^{2 p}+\left|Z_{s}^{t, x}\right|^{2}\right) I_{\mathcal{K}}(s, x) \rho^{-1}(x) d x d s\right]<\infty .
\end{aligned}
$$

Thus, we can apply Lebesgue's dominated convergence theorem to the following calculation:

$$
\begin{aligned}
& \lim _{n \rightarrow \infty} E\left[\int_{t}^{T} \int_{\mathbb{R}^{d}}\left|f_{n}\left(s, X_{s}^{t, x}, Y_{s}^{t, x, n}, Z_{s}^{t, x, n}\right) I_{\mathcal{K}}(s, x)-f\left(s, X_{s}^{t, x}, Y_{s}^{t, x}, Z_{s}^{t, x}\right) I_{\mathcal{K}}(s, x)\right|^{2} \rho^{-1}(x) d x d s\right] \\
= & E\left[\int_{t}^{T} \int_{\mathbb{R}^{d}} \lim _{n \rightarrow \infty}\left|f_{n}\left(s, X_{s}^{t, x}, Y_{s}^{t, x, n}, Z_{s}^{t, x, n}\right)-f\left(s, X_{s}^{t, x}, Y_{s}^{t, x}, Z_{s}^{t, x}\right)\right|^{2} I_{\mathcal{K}}(s, x) \rho^{-1}(x) d x d s\right] \\
\leq & 2 E\left[\int_{t}^{T} \int_{\mathbb{R}^{d}} \lim _{n \rightarrow \infty}\left|f_{n}\left(s, X_{s}^{t, x}, Y_{s}^{t, x, n}, Z_{s}^{t, x, n}\right)-f\left(s, X_{s}^{t, x}, Y_{s}^{t, x, n}, Z_{s}^{t, x, n}\right)\right|^{2} I_{\mathcal{K}}(s, x) \rho^{-1}(x) d x d s\right] \\
& +2 E\left[\int_{t}^{T} \int_{\mathbb{R}^{d}} \lim _{n \rightarrow \infty}\left|f\left(s, X_{s}^{t, x}, Y_{s}^{t, x, n}, Z_{s}^{t, x, n}\right)-f\left(s, X_{s}^{t, x}, Y_{s}^{t, x}, Z_{s}^{t, x}\right)\right|^{2} I_{\mathcal{K}}(s, x) \rho^{-1}(x) d x d s\right] .
\end{aligned}
$$

Since $Y_{s}^{t, x, n} \longrightarrow Y_{s}^{t, x}$ for a.a. $s \in[t, T], x \in \mathbb{R}^{d}$ a.s., there exists a $N(s, x, \omega)$ s.t. when $n \geq N(s, x, \omega)$, $\left|Y_{s}^{t, x, n}\right| \leq\left|Y_{s}^{t, x}\right|+1$. So taking $n \geq \max \left\{N(s, x, \omega),\left|Y_{s}^{t, x}\right|+1\right\}$, we have $f_{n}\left(s, X_{s}^{t, x}, Y_{s}^{t, x, n}, Z_{s}^{t, x, n}\right)=$ $f\left(s, X_{s}^{t, x}, \frac{\inf \left(n,\left|Y_{s}^{t, x, n}\right|\right)}{\left|Y_{s}^{t, x, n}\right|} Y_{s}^{t, x, n}, Z_{s}^{t, x, n}\right)=f\left(s, X_{s}^{t, x}, Y_{s}^{t, x, n}, Z_{s}^{t, x, n}\right)$. That is to say

$\lim _{n \rightarrow \infty}\left|f_{n}\left(s, X_{s}^{t, x}, Y_{s}^{t, x, n}, Z_{s}^{t, x, n}\right)-f\left(s, X_{s}^{t, x}, Y_{s}^{t, x, n}, Z_{s}^{t, x, n}\right)\right|^{2}=0$ for a.a. $s \in[t, T], x \in \mathbb{R}^{d}$ a.s. On the other hand, $\lim _{n \rightarrow \infty}\left|f\left(s, X_{s}^{t, x}, Y_{s}^{t, x, n}, Z_{s}^{t, x, n}\right)-f\left(s, X_{s}^{t, x}, Y_{s}^{t, x}, Z_{s}^{t, x}\right)\right|^{2}=0$ for a.a. $s \in[t, T], x \in \mathbb{R}^{d}$ a.s. is obvious due to the continuity of $(y, z) \rightarrow f(s, x, y, z)$.

Therefore by (4.8), $f_{n}\left(s, X_{s}^{t, x}, Y_{s}^{t, x, n}, Z_{s}^{t, x, n}\right) I_{\mathcal{K}}(s, x)=U_{s}^{t, x, n} I_{\mathcal{K}}(s, x)$ converges strongly to $f\left(s, X_{s}^{t, x}, Y_{s}^{t, x}, Z_{s}^{t, x}\right) I_{\mathcal{K}}(s, x)$ in $L_{\rho}^{2}\left(\Omega \times[t, T] \times \mathbb{R}^{d} ; \mathbb{R}^{1}\right)$, but $U_{s}^{t, x, n} I_{\mathcal{K}}(s, x)$ converges weakly to $U_{s}^{t, x} I_{\mathcal{K}}(s, x)$ in $L_{\rho}^{2}\left(\Omega \times[t, T] \times \mathbb{R}^{d} ; \mathbb{R}^{1}\right)$, so $f\left(s, X_{s}^{t, x}, Y_{s}^{t, x}, Z_{s}^{t, x}\right) I_{\mathcal{K}}(s, x)=U_{s}^{t, x} I_{\mathcal{K}}(s, x)$ for a.a. $r \in[t, T], x \in \mathbb{R}^{d}$ a.s. The lemma follows when $K \rightarrow \infty$.

Proof of Theorem 2.4. With Proposition 4.7 and Lemma 4.8 the existence of solutions to BSDE $(2.2)$ is easy to see. Now we prove the uniqueness. If there is another solution $\left(\tilde{Y}_{s}^{t, x}, \tilde{Z}_{s}^{t, x}\right)$ to BSDE (2.2), then for a.a. $x \in \mathbb{R}^{d},\left(Y_{s}^{t, x}-\tilde{Y}_{s}^{t, x}, Z_{s}^{t, x}-\tilde{Z}_{s}^{t, x}\right)$ satisfies

$$
Y_{s}^{t, x}-\tilde{Y}_{s}^{t, x}=\int_{s}^{T}\left(f\left(r, X_{r}^{t, x}, Y_{r}^{t, x}, Z_{r}^{t, x}\right)-f\left(r, X_{r}^{t, x}, \tilde{Y}_{r}^{t, x}, \tilde{Z}_{r}^{t, x}\right)\right) d r-\int_{s}^{T}\left\langle Z_{r}^{t, x}-\tilde{Z}_{r}^{t, x}, d W_{r}\right\rangle .
$$

Applying Itô's formula to $\left|Y_{s}^{t, x}-\tilde{Y}_{s}^{t, x}\right|^{2}$, by the stochastic Fubini theorem and Conditions (H.3) ${ }^{*}$ and (H.4), we have

$$
E\left[\int_{\mathbb{R}^{d}}\left|Y_{s}^{t, x}-\tilde{Y}_{s}^{t, x}\right|^{2} \rho^{-1}(x) d x\right]+E\left[\int_{s}^{T} \int_{\mathbb{R}^{d}}\left|Z_{r}^{t, x}-\tilde{Z}_{r}^{t, x}\right|^{2} \rho^{-1}(x) d x d r\right]
$$




$$
\leq 2 L^{2} E\left[\int_{s}^{T} \int_{\mathbb{R}^{d}}\left|Y_{r}^{t, x}-\tilde{Y}_{r}^{t, x}\right|^{2} \rho^{-1}(x) d x d r\right]+\frac{1}{2} E\left[\int_{s}^{T} \int_{\mathbb{R}^{d}}\left|Z_{r}^{t, x}-\tilde{Z}_{r}^{t, x}\right|^{2} \rho^{-1}(x) d x d r\right] .
$$

By Gronwall's inequality, the uniqueness of the solution to BSDE (2.2) follows immediately.

By the stochastic flow $X_{r}^{s, X_{s}^{t, x}}=X_{r}^{t, x}$ for $t \leq s \leq r \leq T$ and the uniqueness of solution of BSDE (2.2), following a similar argument as Proposition 3.4 in [25] we have

Corollary 4.9 Under the conditions of Theorem 2.4. let $\left(Y_{s}^{t, x}, Z_{s}^{t, x}\right)$ be the solution of BSDE (2.2), then

$$
Y_{s}^{t, x}=Y_{s}^{s, X_{s}^{t, x}}, Z_{s}^{t, x}=Z_{s}^{s, X_{s}^{t, x}} \text { for any } s \in[t, T] \text {, a.a. } x \in \mathbb{R}^{d} \text { a.s. }
$$

\section{The PDEs}

Now we make use of the results for BSDE (2.2) to give the probabilistic representation to PDEs with p-growth coefficients. Actually the solution of BSDE in the $\rho$-weighted $L^{2}$ space gives the unique weak solution of its corresponding PDE (2.4).

Proof of Theorem 2.6. Using Corollary 4.5, we first prove the relationship between $(Y, Z)$ and $u$, when we take $u(t, x)=Y_{t}^{t, x}$. Having proved Lemma 4.6, we only need to prove that $\left(\sigma^{*} \nabla u\right)\left(s, X_{s}^{t, x}\right)=Z_{s}^{t, x}$ for a.a. $s \in[t, T], x \in \mathbb{R}^{d}$ a.s. This can be deduced from Corollary 4.9 and the strong convergence of $Z^{t, \cdot, n}$ to $Z^{t, \cdot}$ in $L^{2}\left(\Omega \times[t, T] ; L_{\rho}^{2}\left(\mathbb{R}^{d} ; \mathbb{R}^{1}\right)\right)$ by the similar argument as in Proposition 4.2 in [25].

We then prove that $u(t, x)$ defined above is the unique weak solution of PDE (2.4). We still start from PDE (3.3). Let $u^{n}(s, x)$ be the weak solution of PDE (3.3). Then by the definition for the weak solution of PDE, we know $\left(u_{n}, \sigma^{*} \nabla u_{n}\right) \in L^{2}\left([0, T] ; L_{\rho}^{2}\left(\mathbb{R}^{d} ; \mathbb{R}^{1}\right)\right) \times L^{2}\left([0, T] ; L_{\rho}^{2}\left(\mathbb{R}^{d} ; \mathbb{R}^{d}\right)\right)$ and for an arbitrary $\varphi \in C_{c}^{\infty}\left(\mathbb{R}^{d} ; \mathbb{R}^{1}\right)$,

$$
\begin{aligned}
& \int_{\mathbb{R}^{d}} u_{n}(t, x) \varphi(x) d x-\int_{\mathbb{R}^{d}} u_{n}(T, x) \varphi(x) d x-\frac{1}{2} \int_{t}^{T} \int_{\mathbb{R}^{d}}\left(\left(\sigma^{*} \nabla u_{n}\right)(s, x)\right)^{*}\left(\sigma^{*} \nabla \varphi\right)(x) d x d s \\
& -\int_{t}^{T} \int_{\mathbb{R}^{d}} u_{n}(s, x) \operatorname{div}((b-\tilde{A}) \varphi)(x) d x d s \\
= & \int_{t}^{T} \int_{\mathbb{R}^{d}} f_{n}\left(s, x, u_{n}(s, x),\left(\sigma^{*} \nabla u_{n}\right)(s, x)\right) \varphi(x) d x d s .
\end{aligned}
$$

We can prove along a subsequence that each term of (5.1) converges to the corresponding term of (2.5). By (4.5), we know that $u_{n}$ converges strongly to $u$ in $L_{\rho}^{2}\left([0, T] \times \mathbb{R}^{d} ; \mathbb{R}^{1}\right)$, thus $u_{n}$ also converges weakly. Moreover, $\sup _{x \in \mathbb{R}^{d}}(|\operatorname{div}((b-\tilde{A}) \varphi)(x)|)<\infty$ and $\rho$ is a continuous function in $\mathbb{R}^{d}$, so it is obvious that

$$
\lim _{n \rightarrow \infty} \int_{t}^{T} \int_{\mathbb{R}^{d}} u_{n}(s, x) \operatorname{div}((b-\tilde{A}) \varphi)(x) d x d s=\int_{t}^{T} \int_{\mathbb{R}^{d}} u(s, x) \operatorname{div}((b-\tilde{A}) \varphi)(x) d x d s .
$$

Also it is easy to see that

$$
\lim _{n \rightarrow \infty} \frac{1}{2} \int_{t}^{T} \int_{\mathbb{R}^{d}}\left(\left(\sigma^{*} \nabla u_{n}\right)(s, x)\right)^{*}\left(\sigma^{*} \nabla \varphi\right)(x) d x d s
$$




$$
\begin{aligned}
& =\lim _{n \rightarrow \infty}-\frac{1}{2} \int_{t}^{T} \int_{\mathbb{R}^{d}} u_{n}(s, x) \operatorname{div}\left(\sigma \sigma^{*} \nabla \varphi\right)(x) \rho(x) \rho^{-1}(x) d x d s \\
& =-\frac{1}{2} \int_{t}^{T} \int_{\mathbb{R}^{d}} u(s, x) \operatorname{div}\left(\sigma \sigma^{*} \nabla \varphi \sigma\right)(x) \rho(x) \rho^{-1}(x) d x d s \\
& =\frac{1}{2} \int_{t}^{T} \int_{\mathbb{R}^{d}}\left(\left(\sigma^{*} \nabla u\right)(s, x)\right)^{*}\left(\sigma^{*} \nabla \varphi\right)(x) d x d s .
\end{aligned}
$$

Also we have proved that $f_{n}\left(s, X_{s}^{t, x}, Y_{s}^{t, x, n}, Z_{s}^{t, x, n}\right)$ converges weakly to $f\left(s, X_{s}^{t, x}, Y_{s}^{t, x}, Z_{s}^{t, x}\right)$ in $L_{\rho}^{2}(\Omega \times$ $\left.[t, T] \times \mathbb{R}^{d} ; \mathbb{R}^{1}\right)$. In fact we can follow the same procedure as in the proof of Lemma 4.8 to prove $f_{n}\left(s, x, u_{n}(s, x),\left(\sigma^{*} \nabla u_{n}\right)(s, x)\right)$ converges weakly to $f\left(s, x, u(s, x),\left(\sigma^{*} \nabla u\right)(s, x)\right)$ in $L_{\rho}^{2}\left([t, T] \times \mathbb{R}^{d} ; \mathbb{R}^{1}\right)$. So we have

$$
\begin{aligned}
& \lim _{n \rightarrow \infty} \int_{t}^{T} \int_{\mathbb{R}^{d}} f_{n}\left(s, x, u_{n}(s, x),\left(\sigma^{*} \nabla u_{n}\right)(s, x)\right) \varphi(x) d x d s \\
= & \int_{t}^{T} \int_{\mathbb{R}^{d}} f\left(s, x, u(s, x),\left(\sigma^{*} \nabla u\right)(s, x)\right) \varphi(x) d x d s .
\end{aligned}
$$

For any $t \in[0, T], \lim _{n \rightarrow \infty} \int_{\mathbb{R}^{d}} u_{n}(t, x) \varphi(x) d x=\int_{\mathbb{R}^{d}} u(t, x) \varphi(x) d x$ can be proved as follows using Corollary 4.5

$$
\begin{aligned}
\lim _{n \rightarrow \infty}\left|\int_{\mathbb{R}^{d}}\left(u_{n}(t, x)-u(t, x)\right) \varphi(x) d x\right|^{2} & \leq \lim _{n \rightarrow \infty} C_{p} E\left[\int_{\mathbb{R}^{d}}\left|u_{n}\left(t, X_{t}^{0, x}\right)-u\left(t, X_{t}^{0, x}\right)\right|^{2} \rho^{-1}(x) d x\right] \\
& \leq \lim _{n \rightarrow \infty} C_{p} E\left[\sup _{0 \leq t \leq T} \int_{\mathbb{R}^{d}}\left|Y_{t}^{0, x, n}-Y_{t}^{0, x}\right|^{2} \rho^{-1}(x) d x\right]=0 .
\end{aligned}
$$

Here the convergence in the $S^{2 p}$ space gives us a strong result about the convergence of $\int_{\mathbb{R}^{d}} u_{n}(t, x) \varphi(x) d x$ $\longrightarrow \int_{\mathbb{R}^{d}} u(t, x) \varphi(x) d x$ uniformly in $t$ as $n \rightarrow \infty$. Therefore we can prove (2.5) is satisfied for all $t \in[0, T]$. That is to say $u(t, x)$ is a weak solution of PDE (2.4).

The uniqueness of PDE (2.4) can be derived from the uniqueness of BSDE (2.2). Let $u$ be a solution of PDE (2.4). Define $F(s, x)=f\left(s, x, u(s, x),\left(\sigma^{*} \nabla u\right)(s, x)\right)$. Since $u$ is a solution, so $\int_{0}^{T} \int_{\mathbb{R}^{d}}\left(|u(s, x)|^{2 p}+\right.$ $\left.\left|\left(\sigma^{*} \nabla u\right)(s, x)\right|^{2}\right) \rho^{-1}(x) d x d s<\infty$ and

$$
\begin{aligned}
& \int_{0}^{T} \int_{\mathbb{R}^{d}}|F(s, x)|^{2} \rho^{-1}(x) d x d s \\
\leq & C_{p} \int_{0}^{T} \int_{\mathbb{R}^{d}}\left(\left|f_{0}(s, x)\right|^{2}+|u(s, x)|^{2 p}+\left|\left(\sigma^{*} \nabla u\right)(s, x)\right|^{2}\right) \rho^{-1}(x) d x d s<\infty .
\end{aligned}
$$

Then we get a PDE with the generator $F \in L^{2}\left([0, T] ; L_{\rho}^{2}\left(\mathbb{R}^{d} ; \mathbb{R}^{1}\right)\right)$. For this generator $F$, we claim that $\left(Y_{s}^{t, x}, Z_{s}^{t, x}\right) \triangleq\left(u\left(s, X_{s}^{t, x}\right),\left(\sigma^{*} \nabla u\right)\left(s, X_{s}^{t, x}\right)\right)$ solves the following linear BSDE for a.a. $x \in \mathbb{R}^{d}$ with probability one:

$$
Y_{s}^{t, x}=h\left(X_{T}^{t, x}\right)+\int_{s}^{T} F\left(r, X_{r}^{t, x}\right) d r-\int_{s}^{T}\left\langle Z_{r}^{t, x}, d W_{r}\right\rangle .
$$

First we use the mollifier to smootherize $h$ and $F$, then we get two smootherized sequences $h^{m}$ and $F^{m}$ such that $h^{m}(\cdot) \longrightarrow h(\cdot)$ and $F^{m}(s, \cdot) \longrightarrow F(s, \cdot)$ in $L_{\rho}^{2}\left(\mathbb{R}^{d} ; \mathbb{R}^{1}\right)$ respectively. Denote by $u_{m}(t, x)$ the solution of $\mathrm{PDE}$ on $[0, T]$ with terminal value $h^{m}(x)$ and generator $F^{m}(s, x)$ and by $\left(Y_{s, m}^{t, x}, Z_{s, m}^{t, x}\right)$ the solution of BSDE with terminal value $h^{m}\left(X_{T}^{t, x}\right)$ and generator $F\left(s, X_{s}^{t, x}\right)$, then 
following classical results of Pardoux and Peng [20, we have $Z_{t, m}^{t, x}=\sigma^{*} \nabla u_{m}(t, x)$, and $Y_{s, m}^{t, x}=$ $u_{m}\left(s, X_{s}^{t, x}\right)=Y_{s, m}^{s, X_{i}^{t, x}}, Z_{s, m}^{t, x}=\sigma^{*} \nabla u_{m}\left(s, X_{s}^{t, x}\right)=Z_{s, m}^{s, X_{s}^{t, x}}$. But by standard estimates $\left(Y_{s, m}^{t, x}, Z_{s, m}^{t, x}\right)$ is a Cauchy sequence in $L^{2}\left(\Omega \times[t, T] ; L_{\rho}^{2}\left(\mathbb{R}^{d} ; \mathbb{R}^{1}\right)\right) \times L^{2}\left(\Omega \times[t, T] ; L_{\rho}^{2}\left(\mathbb{R}^{d} ; \mathbb{R}^{d}\right)\right)$. By equivalence of norm principle, $u_{m}(s, x)$ is also a Cauchy sequence in $\mathcal{H}$, where $\mathcal{H}$ is the set of random fields $\left\{w(s, x) ; s \in[0, T], x \in \mathbb{R}^{d}\right\}$ such that $\left(w, \sigma^{*} \nabla w\right) \in L^{2}\left([0, T] ; L_{\rho}^{2}\left(\mathbb{R}^{d} ; \mathbb{R}^{1}\right)\right) \times L^{2}\left([0, T] ; L_{\rho}^{2}\left(\mathbb{R}^{d} ; \mathbb{R}^{d}\right)\right)$ with the norm $\sqrt{E\left[\int_{0}^{T} \int_{\mathbb{R}^{d}}\left(|w(s, x)|^{2}+\left|\left(\sigma^{*} \nabla\right) w(s, x)\right|^{2}\right) \rho^{-1}(x) d x d s\right)}<\infty$. So there exists $u \in \mathcal{H}$ such that $\left(u_{m}, \sigma^{*} \nabla u_{m}\right) \rightarrow\left(u, \sigma^{*} \nabla u\right)$ in $L^{2}\left([0, T] ; L_{\rho}^{2}\left(\mathbb{R}^{d} ; \mathbb{R}^{1}\right)\right) \times L^{2}\left([0, T] ; L_{\rho}^{2}\left(\mathbb{R}^{d} ; \mathbb{R}^{d}\right)\right)$ due to the completeness of $\mathcal{H}$. By the equivalence of norm principle again, we know that $\left(Y_{s}^{t, x}, Z_{s}^{t, x}\right) \triangleq\left(u\left(s, X_{s}^{t, x}\right),\left(\sigma^{*} \nabla u\right)\left(s, X_{s}^{t, x}\right)\right)$ is the limit of Cauchy sequence of $\left(Y_{s, m}^{t, x}, Z_{s, m}^{t, x}\right)$. Now it is easy to pass the limit as $m \rightarrow \infty$ on the BSDE which $\left(Y_{s, m}^{t, x}, Z_{s, m}^{t, x}\right)$ satisfies and conclude that $\left(Y_{s}^{t, x}, Z_{s}^{t, x}\right)$ is a solution of BSDE (5.3).

Noting the definition of $F(s, x), Y_{s}^{t, x}$ and $Z_{s}^{t, x}$, we have that $\left(Y_{s}^{t, x}, Z_{s}^{t, x}\right)$ solves BSDE (2.2) for a.a. $x \in \mathbb{R}^{d}$ with probability one. Moreover, by Lemma 3.2

$$
\begin{aligned}
& E\left[\int_{t}^{T} \int_{\mathbb{R}^{d}}\left(\left|Y_{s}^{t, x}\right|^{2 p}+\left|Z_{s}^{t, x}\right|^{2}\right) \rho^{-1}(x) d x d s\right] \\
\leq & C_{p} \int_{t}^{T} \int_{\mathbb{R}^{d}}|u(s, x)|^{2 p}+\left|\left(\sigma^{*} \nabla u\right)(s, x)\right|^{2} \rho^{-1}(x) d x d s<\infty .
\end{aligned}
$$

As Proposition 4.7, we can further deduce that $Y^{t, \cdot} \in S^{2 p}\left([t, T] ; L_{\rho}^{2 p}\left(\mathbb{R}^{d} ; \mathbb{R}^{1}\right)\right)$ and therefore $\left(Y_{s}^{t, x}, Z_{s}^{t, x}\right)$ is a solution of BSDE (2.2). If there is another solution $\hat{u}$ to PDE (2.4), then by the same procedure, we can find another solution $\left(\hat{Y}_{s}^{t, x}, \hat{Z}_{s}^{t, x}\right)$ to BSDE (2.2), where

$$
\hat{Y}_{s}^{t, x}=\hat{u}\left(s, X_{s}^{t, x}\right) \text { and } \hat{Z}_{s}^{t, x}=\left(\sigma^{*} \nabla \hat{u}\right)\left(s, X_{s}^{t, x}\right) .
$$

By Theorem 2.4, the solution of BSDE (2.2) is unique. Therefore

$$
Y_{s}^{t, x}=\hat{Y}_{s}^{t, x} \text { for a.a. } s \in[t, T] \text {, a.a. } x \in \mathbb{R}^{d} \text { a.s. }
$$

In particular, when $t=0$,

$$
Y_{s}^{0, x}=\hat{Y}_{s}^{0, x} \text { for a.a. } s \in[0, T], x \in \mathbb{R}^{d} \text { a.s. }
$$

By Lemma 3.2 again,

$$
\left.\int_{0}^{T} \int_{\mathbb{R}^{d}}|u(s, x)-\hat{u}(s, x)|^{2} \rho^{-1}(x) d x d s \leq C_{p} E\left[\int_{0}^{T} \int_{\mathbb{R}^{d}}\left|Y_{s}^{0, x}-\hat{Y}_{s}^{0, x}\right|^{2}\right) \rho^{-1}(x) d x d s\right]=0 .
$$

So $u(s, x)=\hat{u}(s, x)$ for a.a. $s \in[0, T], x \in \mathbb{R}^{d}$ a.s. The uniqueness is proved. The uniqueness implies that for any selection $u$ in the equivalence class of solution of the PDE (2.4), $u(s, x)=Y_{s}^{s, x}$ for a.a. $s \in[0, T], x \in \mathbb{R}^{d}$. Moreover, noting that $\left(u\left(s, X_{s}^{t, x}\right), \sigma^{*} \nabla u\left(s, X_{s}^{t, x}\right)\right)$ solves the BSDE (2.2) and using the uniqueness of solution of BSDE (2.2) in the equivalence class, we have (2.6) for any representative $Y$ in the equivalence class of the solution of BSDE (2.2).

Acknowledgements. We would like to acknowledge useful conversations with K.D. Elworthy, C.R. Feng, J. Lorinczi, K.N. Lu, S.G. Peng and S.J. Tang. QZ would like to thank the Department of Mathematical Sciences of Loughborough University for appointing him as a Research Associate. He wishes to acknowledge their financial support to the project through the appointment and partial financial sup- 
port of the National Basic Research Program of China (973 Program) with Grant No.2007CB814904. HZ would like to thank K.N. Lu for inviting him to visit Brigham-Young University under their programme of Special Year on Stochastic Dynamics and S.J. Tang for inviting him to visit Laboratory of Mathematics for Nonlinear Science, Fudan University. We are grateful to the referee for his/her constructive comments.

\section{References}

1. V. Bally, A. Matoussi, Weak solutions for SPDEs and backward doubly stochastic differential equations. J. Theor. Probab., Vol.14 (2001), 125-164.

2. G. Barles, E. Lesigne, SDE, BSDE and PDE. In: Backward stochastic differential equations. Pitman Res. Notes Math., Ser.364, Longman, Harlow, (1997), 47-80.

3. Ph. Briand, Y. Hu, BSDE with quadsatic growth and unbounded terminal value. Probab. Theory Rel., Vol.136 (2006), 604-618.

4. R. C. Dalang, C. Mueller and R. Tribe, A Feynman-Kac-type formula for the deterministic and stochastic wave equations and other P.D.E.'s. Trans. AMS, Vol.360 (2008), 4681-4703.

5. R. W. R. Darling, Constructing Gamma-martingales with prescribed limits, using backward SDE. Ann. Probab., Vol.23 (1995), 1234-1261.

6. M. D. Donsker, S. R. S. Varadhan, Asymptotic evaluation of certain Markov process expectations for large time, I and II. Comm. Pure. Appl. Math., Vol.28 (1975), 1-47 and 279-301.

7. K. D. Elworthy, A. Truman, H. Z. Zhao, J. G. Gaines, Approximate travelling waves for generalized KPP equations and classical mechanics. Proc. R. Soc. London, Sect.A, Vol.446 (1994), 529-554.

8. R. P. Feynman, Space-time approach to non-relativistic quantum mechanics. Rev. Mod. Phys., Vol.20 (1948), 367-387.

9. M. I. Freidlin, Functional integration and partial differential equations. Annals of Mathematics Studies, Vol.109, Princeton University Press, Princeton, New Jersey (1985).

10. K. D. Elworthy, Geometric aspects of diffusions on manifolds. Ecole d'Eté de Probabilités de Saint Flour, XVII. Lecture Notes in Math. 1362 276-425. Springer, Berlin. (1987).

11. N. El-Karoui, S. Peng, M. C. Quenez, Backward stochastic differential equations in finance. Math. Finance, Vol.7 (1997), 1-72.

12. M. Kac, On distributions of certain Wiener functionals. Trans. AMS, Vol.65 (1949), 1-13.

13. M. Kobylanski, Backward stochastic differential equations and partial differential equations with quadratic growth. Ann. Probab., Vol.28 (2000), 558-602.

14. H. Kunita, Stochastic flow acting on Schwartz distributions. J. Theor. Probab., Vol.7 (1994), 247-278.

15. J. P. Lepeltier, J. San Martin, Backward stochastic differential equations with continuous coefficient. Stat. Probab. Lett., Vol.32 (1997), 425-430.

16. P. Malliavin, D. W. Stroock, Short time behaviour of the heat kernel and its logarithmic derivatives. J. Differ. Geom., Vol.44 (1996), 550-570.

17. A. Matoussi and M. Xu, Sobolev solution for semi-linear PDE with obstacle under monotonicity condition. Electron. J. Probab., Vol.13 (2008), 1035-1067.

18. E. Pardoux, BSDE's weak convergence and homogenization of semilinear PDE's. In F. Clarke and R. Stern eds. Nonlin. Analy., Diff. Equa. and Control, Kluwer Acad. Publi. Dordrecht (1999), 503-549.

19. E. Pardoux, S. Peng, Adapted solution of a backward stochastic differential equation. Syst. Control Lett., Vol.14 (1990), 55-61.

20. E. Pardoux, S. Peng, Backward stochastic differential equations and quasilinear parabolic partial differential equations. Stochastic Partial Differential Equations, B. L. Rozuvskii, R. B. Sowers eds., Lect. Notes Control Inf. Sci., Berlin Heidelberg New York: Springer, Vol.176 (1992), 200-217.

21. J. C. Robinson, Infinite-dimensional dynamical systems: an introduction to dissipative parabolic PDEs and the theory of global attractors. Cambridge University Press (2001).

22. B. Simon, Functional integration and quantum physics. AMS Chelsea Publishing, 2nd Edition, (2005).

23. R. Temam, Infinite-dimensional dynamical systems in mechanics and physics. Applied Mathematical Sciences, 68. Springer-Verlag, New York (1988).

24. A. D. Wentzell, M. I. Freidlin, On small random perturbations of dynamical system. Russ. Math. Surv., Vol.25 (1970), 1-55.

25. Q. Zhang, H. Z. Zhao, Stationary solutions of SPDEs and infinite horizon BDSDEs. J. Funct. Anal., Vol.252 (2007), 171-219. 
26. Q. Zhang, H. Z. Zhao, Stationary solutions of SPDEs and infinite horizon BDSDEs with non-Lipschitz coefficients. J. Differ. Equations, Vol.248 (2010), 953-991.

27. Q. Zhang, H. Z. Zhao, SPDEs with Polynomial Growth Coefficients: Weak Solutions via BDSDEs and Stationary Solutions. Preprint. 